\title{
Eine neue Roman-Handschrift.
}

\section{Die Fundgeschichte.}

Als ich im November 1898 im hundertthorigen Theben weilte, wurde mir u. a. ein ganzer Stofs von Pergamentblättern, die den verschiedensten Codices entstammten, zum Kauf angeboten. Da es sämtlich koptische und zwar sehr junge koptische Handschriften waren, wollte ich sie schon bei Seite schieben, als ich auf einem der Blätter unter der dicken, schwarzen Schrift der koptischen Hand eine matte, hellgelbe griechische Uncialschrift entdeckte. Bald fanden sich noch mehrere derartige Blätter hinzu; im ganzen waren es sieben, die ich mir dann käuflich erwarb. Es gehört zu meinen schönsten Reiseerinnerungen, wie ich darauf in meiner Feluke den Nil stromabfahrend mich in das ehrwürdige Pergamen vertiefte und erst einzelne Worte, dann ganze Sätze des griechischen Palimpsestes entzifferte, bis mir die schon bei der ersten Durchsicht in Theben in den Sinn gekommene Vermutung, dals ich einen griechischen Roman vor mir habe, zur Gewifsheit wurde. Bald zogen mich andere Aufgaben von der Handschrift ab. Nur vorübergehend habe ich noch im Zelt zu Abusir und dann in Cairo Mufse gehabt, die Entzifferung des Palimpsestes zu fördern. Wesentlich wurde ich hierbei dank der Liebenswürdigkeit des stets hilfbereiten Herrn Dr. von Bissing dadurch unterstützt, dals er mir seine Didot'sche Ausgabe der Scriptores Erotici lieh. Natürlich mufste ich mich auf die besser erhaltenen Teile des Palimpsestes beschränken und hoffte, später in der Heimat mit Hilfe chemischer Reagentien auch die anderen Partien bezwingen zu können.

Diese Hoffnung sollte nicht in Erfüllung gehen. Durch eine jener unberechenbaren Launen der Tyche, wie sie die griechischen Romanschriftsteller nicht müde wurden zu ersinnen, sind die Pergamentblätter für immer verloren gegangen: auf dem Schiffe, das sie im Frühling 1899 sicher bis an die heimatliche Küste gebracht hatte, brach vor der Ausladung im Hafen von Hamburg Feuer aus, und bei diesem Brande sind sowohl jene Pergamentblätter als auch die Papyri, die ich im Auftrage der Generalverwaltung der königlichen Museen zu Berlin 
vom Januar bis März 1899 in Ehnâs-Herakleopolis mit Heinrich Schaefer zusammen ausgegraben hatte, der Vernichtung anheimgefallen. Was wir durch die Zerstörung der Papyri verloren haben, resp. was durch meine Kopien sich davon für die Wissenschaft noch retten lälst, darüber werde ich ein anderes Mal berichten. Heute beschränke ich mich darauf, mitzuteilen, was ich über jene Pergamentblätter nach meinen Aufzeichnungen und meinen Erinnerungen zu sagen weifs. Wenn diese Mitteilungen lückenhaft sind, so wolle man nicht vergessen, dafs alles, was ich in Ägypten an der Handschrift gethan habe, nur zur vorläufigen Orientierung geschah, der die gründliche Durcharbeitung zu Hause folgen sollte.

\section{Der Codex Thebanus.}

Von den sieben Blättern gehörten sechs zu einem und demselben Codex. Das bewies das gleiche Format, die gleiche Schrift und Schriftanordnung, sowie die Verwandtschaft des Inhalts.

Von diesen sechs Blättern mag jedes nach nachträglicher, rein approximativer Schätzung etwa $20 \mathrm{~cm}$. hoch und $15 \mathrm{~cm}$. breit gewesen sein. Dagegen war das siebente Blatt grölser.

Die griechische Palimpsestschrift jener sechs Blätter war eine feine, sorgsam geschriebene Unciale, die nach rechts hin geneigt war. Gewisse Linien der Schrift liefen in kleine Pünktchen aus. Accente waren nicht vorhanden, ebensowenig Interpunktion oder sonstige Lesezeichen; grölsere Abschnitte wurden vielmehr durch Spatien getrennt. An Abkürzungen habe ich nichts weiter bemerkt als gelegentlich am Schlufs der Zeilen den übergesetzten Horizontalstrich zur Vertretung des schliefsenden $\boldsymbol{\nu}$. Der ganze Ductus erinnerte etwa an die Schrift des Fragmentum Mathematicum Bobiense aus dem VII./VIII. Jahrh. n. Chr. Als ich das Original noch vor mir hatte, habe ich die Schrift des Palimpsestes in das VI. oder VII. Jahrh. n. Chr. gesetzt. Jetzt, nach Vergleichung mancher anderen Texte, ist mir das VII. Jahrh. wahrscheinlicher, doch ist auch das VIII. wohl nicht völlig ausgeschlossen. Noch später dürfte die Handschrift auf keinen Fall anzusetzen sein.

In der Schrift des siebenten Blattes waren die Buchstaben nicht schräg geneigt, sondern standen aufrecht. Die Linien zeigten an ihren Enden dicke Punkte. Ich bin nicht in der Lage, eine genauere zeitliche Schätzung anzugeben. Damals schien mir die Schrift etwas älter als die der sechs Blätter zu sein. Über die Schwierigkeit solcher Datierungen vgl. unten das paläographische Referat. 
Während das siebente Blatt in einer einzigen breiten Kolumne beschrieben war, war die Schrift auf den sechs anderen Blättern in der Weise angeordnet, dafs auf jeder Seite zwei schmale Kolumnen neben einander standen. Die Kolumne bestand hier, wie ich nachträglich meinen Kopien entnehme, überall aus 28 Zeilen. Die Zeile hatte im Durchschnitt 13-17 Buchstaben; doch kommen auch 18 vor, andrerseits auch 12, einmal auch 11 (VII 9). Die Anfänge der Zeilen standen genau unter einander, die Zeilenenden, wie häufig, nicht ganz so genau, doch war auch hier eine Gleichheit wohl beabsichtigt, denn gegen Schlufs der Zeile wurden gelegentlich, wenn mich meine Erinnerung nicht täuscht, die Buchstaben kleiner und enger an einander gerückt; auch diente demselben Zweck der oben erwähnte Horizontalstrich für $\boldsymbol{\nu}$. Über die Liniierung des Pergaments kann ich nichts Sicheres mehr aussagen.

Was endlich den Inhalt der griechischen Texte betrifft, so gehörten vier von jenen sechs gleichartigen Blättern einer Handschrift von Charitons Roman von Chaireas und Kallirrhoë an, zwei der Handschrift eines, wie mir scheint, bisher unbekannten Romans von der schönen Chione. Welchem Litteraturwerk das siebente Blatt angehört hat, kann ich nicht sagen, da ich nur einzelne Worte davon entziffert habe. Ich beschäftige mich daher im Folgenden nur mit jenen Romanhandschriften.

Die oben angeführten Indicien machen es zweifellos, dafs der Chariton-Roman und der Chione-Roman in einem und demselben Codex gestanden haben. Dieser Codex, in dem also mindestens zwei griechische Romane vereinigt waren, scheint der Bibliothek eines der oberägyptischen Klöster angehört $\mathrm{zu}$ haben. So berichtete mir wenigstens der Verkäufer, dals die mir angebotenen koptischen Manuskripte sämtlich aus einem benachbarten Kloster stammten. Diese Annahme palst auch gut zu der Thatsache, dafs die griechischen Texte abgewaschen worden sind, um das wertvolle Pergament zur Aufnahme christlicher religiöser Texte in koptischer Sprache - Schaefer taxierte sie, glaube ich, auf Homilien - zu verwenden. Wann diese Reskription erfolgt ist, kann jetzt, wo das Original verloren ist, nicht mehr ausgemacht werden. Ich habe die koptische Schrift unserer sechs Blätter damals etwa in das X.-XII. Jahrh. gesetzt, kann freilich keine Bürgschaft übernehmen.

Wie mag man dazu gekommen sein, die griechischen Romane zu beseitigen? Wenn man beim Kirchenvater Nikephoros Kallistos liest, wie der Bischof Heliodor von der Provinzialsynode aufgefordert wird, entweder den Roman (den er geschrieben haben soll) zu verbrennen 
oder aus dem Amt zu scheiden ${ }^{1}$ ), so könnte man auf den Gedanken kommen, dafs ein glaubenseifriger Mönch voll heiligen Eifers über die sündige Erotik unserer Romane sie abgewaschen und fromme christliche Homilien darüber geschrieben habe. ${ }^{2}$ ) Diese Möglichkeit wird man nicht leicht bestreiten können. Aber ich könnte mir die Geschichte unserer Handschrift auch anders denken: trotz aller Provinzialsynoden mag man zumal in der abgelegenen Thebais die griechischen Romane auch hinter den Klostermauern mit Vergnügen gelesen haben, und so mag sich auch an unserer Handschrift, die wir etwa dem VII. Jahrh. zugewiesen, noch eine Reihe von Generationen ergötzt haben. Allmählich - vielleicht seit dem X. Jahrhundert - schwand dann die Kenntnis des Griechischen, und für diesen Romanjargon half auch keine "Scala“. So mag man denn schliefslich, nachdem der Romancodex nur noch den Wert eines Schreibstoffes hatte, die koptischen Homilien darüber geschrieben haben.

Ich habe nicht nachgeforscht, ob schon mehrere griechische Palimpseste aus koptischen Klosterbibliotheken bekannt geworden sind. Aber auch schon dieser eine Fund eröffnet uns vielleicht eine erfreuliche Perspektive. Sollte es nicht öfter vorgekommen sein, dafs die griechischen Autoren, die in den Klöstern gelesen wurden, später nach dem Aussterben der griechischen Sprache koptisch reskribiert worden sind? So liefse sich hoffen, dafs vielleicht noch mancher griechische Palimpsest aus koptischen Klosterbibliotheken an's Tageslicht käme!

\section{Der Roman des Chariton.}

Eine neue Handschrift des Chariton wird schon darum überall auf besonderes Interesse rechnen dürfen, weil uns dieser Dichter bekanntlich nur in einer einzigen Handschrift, einem Florentinus aus dem XIII./XIV. Jahrhundert überliefert ist. Das Interesse wird dadurch noch gesteigert, dafs dieser Florentinus besonders schlecht erhalten und sehr schwer lesbar ist, sodals erst nach und nach - namentlich durch Cobet's Verdienst - die Entzifferung gelingen konnte.

Neben diesen Florentinus tritt nun der Codex Thebanus aus dem VII., spätestens VIII. Jahrhundert. Da Chariton nach Rohdes Ansatz

1) Vgl. Rohde, Gr. Rom. S. 432.

2) Nach Rohde wären Achilles Tatius und Chariton Christen gewesen. Erwiesen ist es nicht. - Ich betone, dals diejenigen Schriftsteller, die christlichen Bischöfen die Autorschaft von Romanen zuschreiben, doch hervorheben, dals sie dies gethan, ehe sie Bischöfe, resp. Christen geworden. Vgl. zu Heliodor Rohde S. 432 und zu Achilles Tatius S. 470, Anm. 1. 
erst im V./VI. Jahrhundert gelebt hat, so repräsentiert der Codex Thebanus, der nur 1-2 Jahrhunderte jünger ist, eine recht alte Tradition. Bei der Wichtigkeit der Handschrift hielt ich es nicht für überflüssig, statt einer kurzen Mitteilung der neuen Lesarten vielmehr den ganzen Text des Palimpsestes, soweit ich über ihn verfüge, mitzuteilen, zumal sich gelegentlich erst durch die Beobachtung der Raumverhältnisse Abweichungen zwischen den beiden Handschriften ergeben.

Von den sechs Blättern des Romancodex gehören, wie oben gesagt, vier zum Roman des Chariton. Von diesen vier habe ich bei meiner vorläufigen Entzifferung in Ägypten nur die glatteren, besser erhaltenen und leichter lesbaren Fleischseiten berücksichtigt, während ich die Haarseiten, die mit blofsem Auge oder auch mit der Lupe kaum lesbar waren, für die spätere Behandlung mit Reagentien reservierte. So sind vier Seiten oder acht Kolumnen, also gerade die Hälfte der von mir erworbenen Charitonhandschrift, durch meine Kopie gerettet worden. Es ist ein besonderes Glück, dals ich, wie schon oben bemerkt, dank der Liebenswürdigkeit des Herrn von Bissing diese neue Charitonhandschrift während der Entzifferung mit einer Edition des Romanes vergleichen konnte. Bei den zahlreichen Abweichungen des Codex Thebanus würde ich sonst keine völlige Sicherheit haben, dafs sie wirklich so in der Handschrift stehen und nicht etwa verunglückten Lesungen ihr Dasein verdanken. Nun aber habe ich schon während der Entzifferung die Abweichungen von der Tradition kennen gelernt und habe im Bewufstsein der Verschiedenheit die divergierenden Stellen mit doppelter Aufmerksamkeit prüfen können. Ich kann daher mit gutem Gewissen die Fachgenossen bitten, der folgenden Kopie der acht Kolumnen ihr Vertrauen entgegen zu bringen, wenn auch die Richtigkeit am Original nicht mehr nachgeprüft werden kann.

Nur an wenigen Stellen sind mir Bedenken über die Richtigkeit meiner Kopie gekommen. Die wenigen Buchstaben, die ich nachträglich vermutungsweise geändert habe, sind in spitze Klammern \langle\rangle eingeschlossen. Alles andere wird so mitgeteilt, wie ich es am Original gelesen habe, mit allen Eigentümlichkeiten und Fehlem des Originals, unter Hinzufügung von Accenten, Spiritus, Jota subscripta und Interpunktion. Die Lücken sind nach dem Florentinus ergänzt, soweit nach Malsgabe des Raumes Übereinstimmung der Traditionen anzunehmen war. Rechts neben dem Thebanus habe ich die abweichenden Lesungen des Florentinus notiert. Die entsprechenden Wörter in Th. sind fett gedruckt.

Da die vier Blätter unmittelbar hinter einander gehörten und, wie Gregory gezeigt, die Lagen des Codex so geordnet zu werden pflegten, 
dafs immer Fleischseite neben Fleischseite und Haarseite neben Haarseite zu stehen kam, so ergiebt sich folgende Anordnung des Handschriftenrestes:

1. Blatt Haarseite: nicht kopiert.

$\left.\begin{array}{l}\text { Fleischseite: Col. I und II } \\ \text { Fleischseite: Col. III und IV }\end{array}\right\}=$ Char. VIII 5, 9-6, 1.

2. Blatt Fleischseite: Col. III und IV
Haarseite: nicht kopiert

3. Blatt Haarseite: nicht kopiert. Jier stand Char. VIII 6, 1-6, 8 .

$\left.\begin{array}{l}\text { Fleischseite: Col. V und VI } \\ \text { Fleischseite: Col. VII und VIII }\end{array}\right\}=$ Char. 6, 8-7,3.

Haarseite: nicht kopiert.

Codex Thebanus.

Codex Florentinus.

Kolumne I.

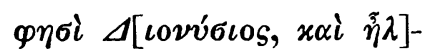

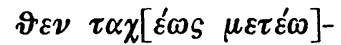

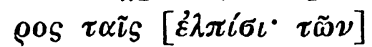

$[\gamma \dot{\alpha} \varrho \pi \varepsilon \rho l \quad X] \alpha \iota \varepsilon^{\alpha} \alpha \nu[o ̛ ̉]-$

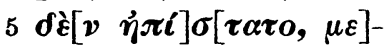

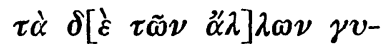

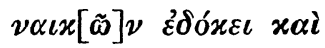

$K \alpha \lambda \lambda \iota \varrho o ́ \eta \nu \pi \alpha \rho \varepsilon \tilde{\nu} \alpha \iota$

$x \alpha i \quad \beta \alpha \sigma \iota \lambda \varepsilon \dot{\alpha} \alpha[\alpha \lambda \varepsilon \bar{\tau}] \nu \quad \alpha \dot{v}-$

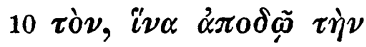

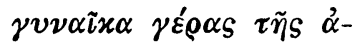

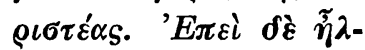

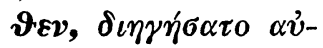

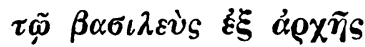

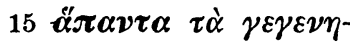

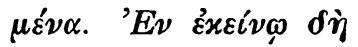

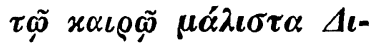

ovv์

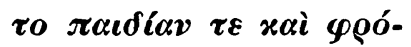

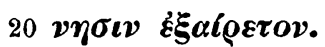

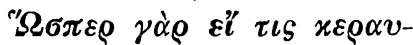

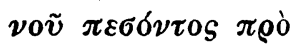

$\tau \tilde{\omega} \nu \pi 0 \delta \tilde{\omega} \nu \alpha \tilde{v} \tau 0 \tilde{v}$

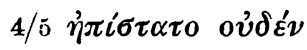

7 xai fehlt.

$12 / 13 \delta^{\prime} \varepsilon i \varsigma \tilde{\eta} \tilde{\lambda} \lambda \varepsilon \varepsilon \nu$

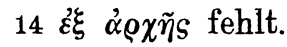

$15 \pi \alpha^{\prime} \nu \tau \alpha$

$16 \delta \dot{\varepsilon}$

$17 \mu \alpha^{\prime} \lambda \iota \sigma \tau \alpha$ fehlt.

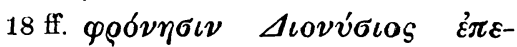
$\delta \varepsilon i \xi \alpha \tau 0 \quad x \alpha i \quad \pi \alpha \iota \delta \varepsilon i \alpha \nu$ हैं $\xi^{\prime} i^{\prime}$

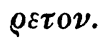

21 Ei fehlt. 
Codex Thebanus.

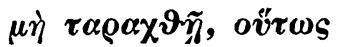

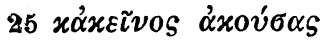

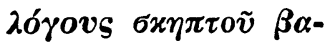

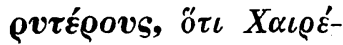

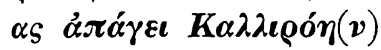

Codex Florentinus.

$24 \tau \alpha \varrho \alpha \chi \vartheta \varepsilon i \eta \eta$. Es fehlt ov́r ergänzt von Hercher.

$26 \lambda \delta \gamma \omega \nu-\beta \alpha \rho v \tau \varepsilon \dot{\rho} \omega \nu$

28 f. $K \alpha \lambda \lambda \iota \varrho \varrho o ́ \eta \nu$ sis $\Sigma v \varrho \alpha \varkappa o v ́ \sigma \alpha \varsigma$ $\dot{\alpha} \pi \alpha \dot{\alpha} \gamma \varepsilon \iota$

\section{Kolumne II.}

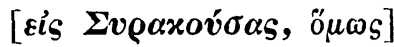

$\left[\begin{array}{lll}\varepsilon \dot{v} \sigma \tau \alpha \vartheta \eta े & \ddot{\varepsilon} \mu \varepsilon \nu \nu \varepsilon & x \alpha i\end{array}\right]$

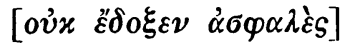

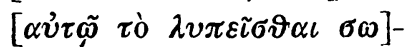

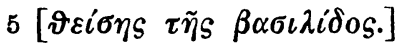

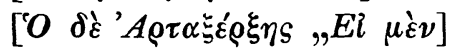

$\dot{\varepsilon} \delta v\left[\nu \alpha ́ \mu \eta \nu, z^{\prime} \varphi \eta ?\right]$

$x \alpha \grave{i} K[\alpha \lambda \lambda \iota \rho o ́ \eta v \dot{\alpha} \pi \dot{\varepsilon}]-$

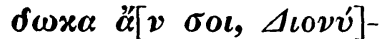

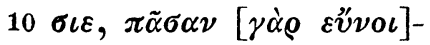

$\alpha \nu x \alpha i \quad \pi i \sigma \tau \iota \nu[\dot{z} \pi \varepsilon]-$

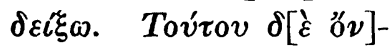

$\tau$ os ảovvátov, $\delta i \delta \omega-$

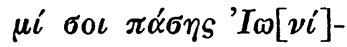

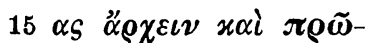

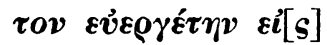

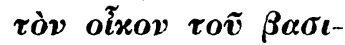

$\lambda \hat{\varepsilon} \omega \subseteq \dot{\alpha} v \alpha \gamma \varrho \alpha \varphi \tilde{\eta} v \alpha \iota$.

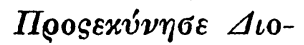

$20 \nu v ́ \sigma \iota 0 s \quad x[\alpha i] \chi \alpha \dot{\alpha}\langle\langle\nu\rangle \delta-$

$\mu \circ \lambda \sigma \gamma \eta^{\prime} \sigma \alpha_{S}[" \tilde{\varepsilon} \sigma \pi \varepsilon v]-$

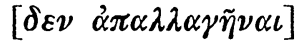

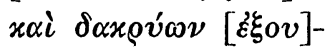

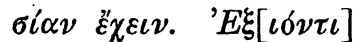

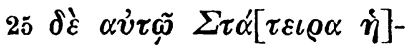

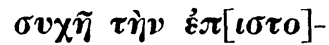

$\lambda \grave{\eta} v \dot{\varepsilon} \pi \iota \delta[\langle i \delta \omega \sigma \iota v]$.

' $\Upsilon \pi 0 \sigma \tau \rho \varepsilon ́ \psi\left[\begin{array}{ll}\alpha \varsigma & \delta \xi\end{array}\right]$

8 xai fehlt.

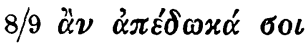

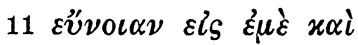

$13 \dot{\alpha} \mu \eta \chi \dot{\alpha} \nu 0 v$

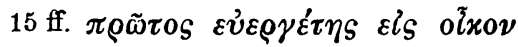

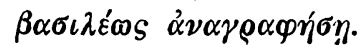

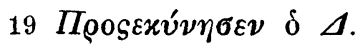

$21 \ddot{z}^{\prime} \chi \varepsilon \iota \nu$ hinter $\delta \mu \circ \lambda \sigma \gamma \eta^{\prime} \sigma \alpha \alpha_{S}$, von Hercher getilgt.

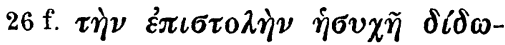
$\sigma \iota \nu$.

II 1-7 zu den Ergänzungen vgl. unten S. 243. - 20 meine Kopie hat $\chi \alpha \rho \varepsilon$,

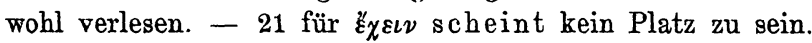


Codex Thebanus.

Codex Florentinus.

Kolumne III.

$\left[\begin{array}{lll}x \alpha i & x \alpha \alpha x \lambda \varepsilon i \sigma \alpha S & \varepsilon\end{array}\right]-$ $[\alpha v \tau \dot{\nu} \nu \gamma \nu] \omega \varrho i \sigma \alpha S \quad \tau \dot{\alpha}$

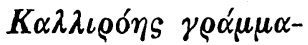
$\tau \alpha \pi \rho \tilde{\tau} \tau 0 \nu \tau \dot{\eta} \nu$ ह́-

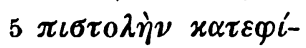
$\lambda \eta \sigma \varepsilon \nu$ xai $\dot{\alpha} \nu o i \xi \alpha S$

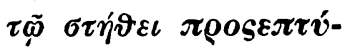
$\xi \alpha \tau o \dot{\omega} s \dot{\varepsilon} x \varepsilon i \nu \eta \nu$ $\pi \alpha \varrho o \tilde{v} \sigma \alpha \nu . \quad ' E \pi \grave{\imath} \quad \pi 0 \lambda \grave{v}(\boldsymbol{v})$

10 d $\alpha \hat{v} \tau \grave{\alpha} \alpha \dot{v} \alpha \gamma \varepsilon t v \omega \sigma \sigma x \iota^{\circ}$

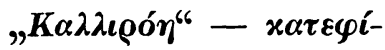

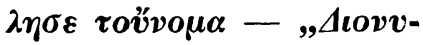

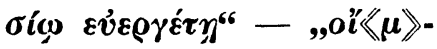

$15 \mu o \imath \tau \tilde{\omega}$ ảvd@í oủx है-

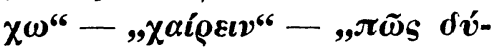

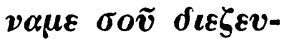

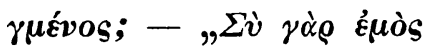

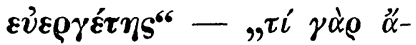

$20 \xi_{\iota \circ \nu} \dot{\pi} \pi \circ i \eta \sigma_{\alpha} \sigma o v$;“

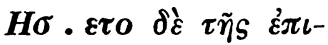

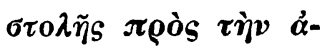

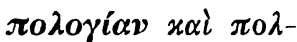
$\left[\lambda \alpha^{\prime}\right] x \iota s \quad \dot{\alpha} \nu \varepsilon \gamma i \nu \omega \sigma x \varepsilon$

$25[\varkappa \alpha ?] \tau \grave{\alpha} \quad \tau \grave{\alpha} \grave{\varrho} \eta^{\prime} \mu \alpha \tau \alpha . \quad$ 'E-

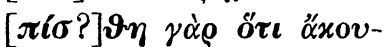
$\left[\begin{array}{ll}\sigma \alpha & x \alpha\end{array}\right] \varepsilon \dot{\lambda} \iota \tau \varepsilon \varepsilon . \quad O \tilde{\tau} \tau \omega$

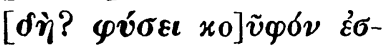

6 عĩ $\alpha$

$7 / 8 \pi \rho \circ \varepsilon \tau i \vartheta \varepsilon$

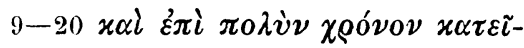
$\chi \varepsilon \nu, \dot{\alpha} \nu \alpha \gamma \iota \nu \omega ́ \sigma x \varepsilon \iota \nu \mu \grave{\eta} \delta v-$

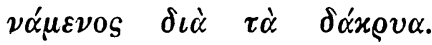

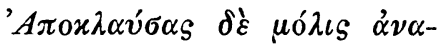

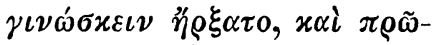

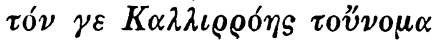

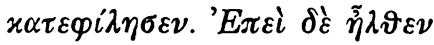

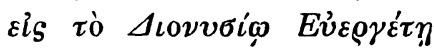

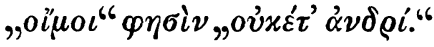

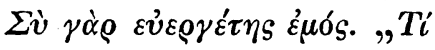

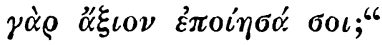

$21^{\prime \prime} H \sigma \vartheta \eta$

22/23 $\tau \tilde{\eta} \dot{\alpha} \pi 0 \lambda \circ \gamma i \alpha$

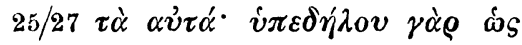

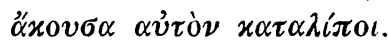

$28[\delta \dot{\eta} \varphi \dot{v} \sigma \varepsilon \iota]$ fehlt.

\section{Kolumne IV.}

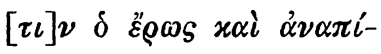

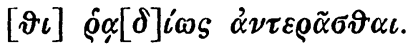
$[\Theta \varepsilon] \alpha \sigma a ́ u \varepsilon v o v$ ờ $\tau \grave{o}$

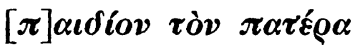

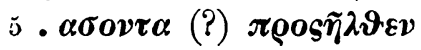

$1 \delta$ auchF, getilgt von Hercher.

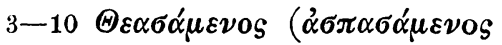

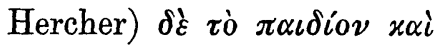

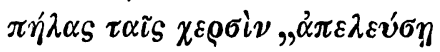

III. 12 ff. die Gedankenstriche, die die Verlesung und die Ausrufe etc. trennen sollen, sind meine Zuthat. - 15/16 zu ov $\xi^{\prime} \chi \omega$ vgl. unten S. 240. 
Codex Thebanus.

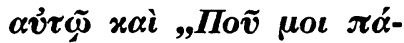

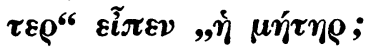

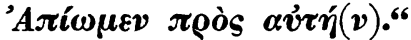

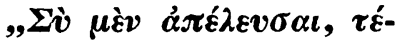

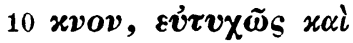

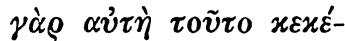

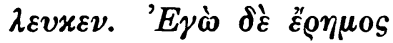
$\beta \iota \dot{\sigma} \sigma 0 \mu \alpha \iota, \pi \alpha \dot{\nu} \nu \tau \omega \nu \alpha i^{\prime}-$

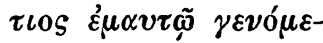

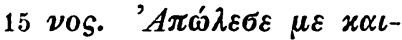

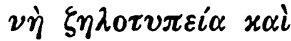

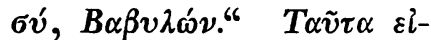
$\pi \dot{\omega} \nu \pi \alpha \varrho \varepsilon \sigma x \varepsilon v \alpha \dot{\zeta} \zeta \varepsilon-$

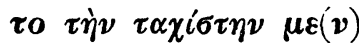

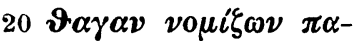

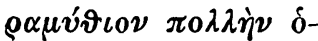

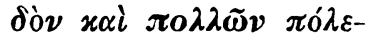
$\omega \nu \dot{\eta} \gamma \varepsilon \mu o v i \alpha \nu$ xai $\tau \grave{\alpha} s$ ย่v $M \varepsilon \iota \lambda \eta^{\prime} \tau \omega K \alpha \lambda-$

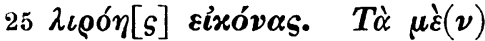

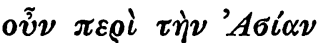

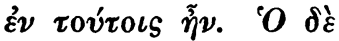

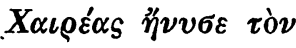

Codex Florentinus.

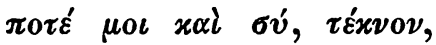
$\pi \varrho \grave{S} \tau \dot{\eta} \nu \mu \eta \tau \varepsilon \dot{\rho} \alpha$

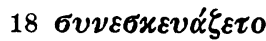

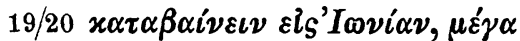

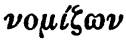

$22 \pi 0 \lambda \lambda \tilde{\omega} \nu$ fehlt; $\pi 0 \lambda \lambda \tilde{\omega} \nu " \partial x \lambda \omega \nu$ Reiske.

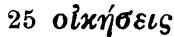

\section{Kolumne $V$.}

$\varphi\llcorner\lambda \alpha \varrho \gamma[v \varrho \ldots . .]$.

$\pi \varepsilon \rho \eta \mu[\ldots \ldots \ldots]$

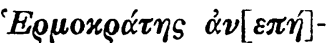

$\delta \eta \sigma \varepsilon \nu \dot{\varepsilon} \pi i \quad \tau \dot{\eta} \nu[\sigma x \eta]-$

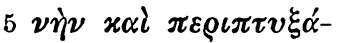

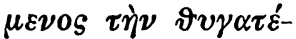

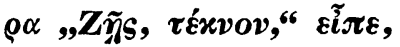

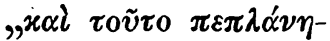

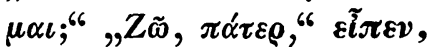

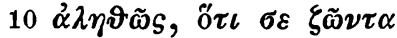
$\tau\left[\varepsilon \vartheta \varepsilon^{\prime} \alpha \mu \alpha \iota\right]{ }^{6} \quad \Delta \alpha^{\prime} x \rho v \alpha \pi \tilde{\alpha}-$ $\sigma \iota \nu \mu \varepsilon\langle\tau\rangle \grave{\alpha}\langle\chi\rangle \alpha\left[\rho \tilde{\alpha}_{S}\right] \dot{\varepsilon} \xi \varepsilon-$
1-2 entspricht in $\mathrm{F}$ nichts.

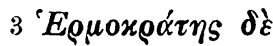

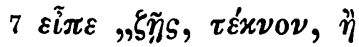

8 xaiauch inF, $v$.Hercher getilgt.

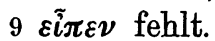

$10 \nu \tilde{v} \nu \dot{\alpha} \lambda \eta \vartheta \tilde{\imath} s$. Es fehlt $\xi \tilde{\omega} \nu \tau \alpha$.

$12 \varepsilon^{\prime} \chi \varepsilon \tilde{\varepsilon} \tau 0 \mu \varepsilon \tau \dot{\alpha} \chi \alpha \rho \tilde{\alpha} s$.

V. 12 meine Kopie hat $\mu \varepsilon \gamma \alpha \lambda \alpha$..., wohl verlesen, denn $\gamma$ ist $\tau$ ähnlich und $\lambda x$.

Archiv f. Papyrusforschung I. 2. 
Codex Thebanus.

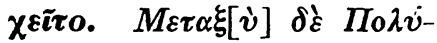
$\chi \alpha \rho \mu \circ \varsigma[\varepsilon \pi \iota x \alpha] \tau \alpha \pi \lambda \varepsilon \tilde{\imath}$

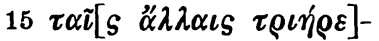

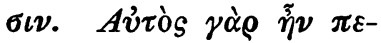

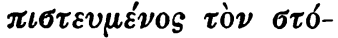

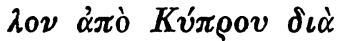

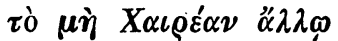

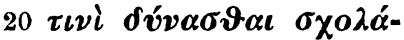

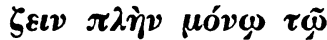

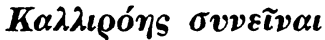

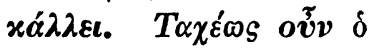

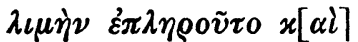

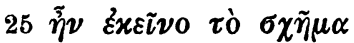
$\tau \grave{o} \mu \varepsilon \tau \dot{\alpha} \tau \grave{\eta} \nu \nu \alpha v \mu \alpha-$

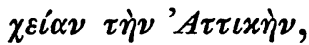

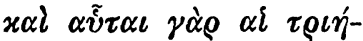

Codex Florentinus.

$15 \tau \alpha \tilde{\varsigma}$ such in $\mathrm{F}$; Hercher: $\sigma \dot{v} \nu$ $\tau \alpha i s$. Dagegen Cobet.

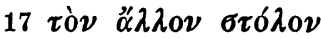

$19 \mu \eta x \varepsilon ́ \tau \iota$

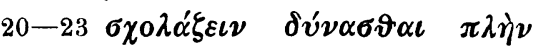

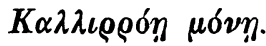

$23 \delta \dot{\varepsilon}$

25 ff. wie F. Vgl. Naber, Mnemosyne N. S. VIII. S. 212; Schmidt,J.Jahrbb.125(1882) S. 194

\section{Kolumne VI.}

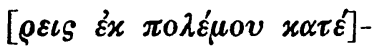
$[\pi \lambda \varepsilon \sigma \nu \varepsilon \sigma \sigma \varepsilon \varepsilon \alpha \nu \omega \mu \varepsilon \dot{\varepsilon}]$ $\left[\begin{array}{lll}\nu \alpha \iota & \varrho \eta \sigma \alpha ́ \mu \varepsilon \nu \alpha \iota & \Sigma v\end{array}\right]$

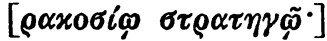

$5\left[\sigma v \nu \varepsilon \mu i^{\prime} \chi \vartheta \eta \sigma \alpha \nu\right.$ हैे $\left.\alpha i\right]$

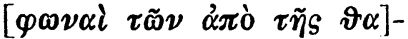

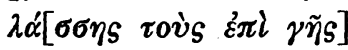
$\dot{\alpha} \sigma \pi \alpha \zeta[o \mu \varepsilon \dot{\nu} \omega \nu, \tau \tilde{\omega \nu}]$

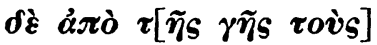

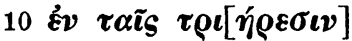
$\varepsilon \dot{v} \varphi \eta \mu i \alpha \iota\left[\begin{array}{lll}\tau \varepsilon & x \alpha \iota & \varepsilon^{\prime} \pi \alpha \iota\end{array}\right]-$

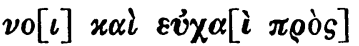

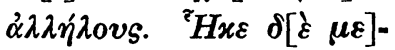
$\tau \alpha \xi \grave{v} \varphi \varepsilon \rho o ́ \mu \varepsilon v o[\mathrm{~s}]$

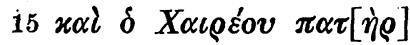

$\lambda \iota \pi 0 \psi v \chi \tilde{\omega} \nu$ ह่ं $\tau[\tilde{\eta} s]$

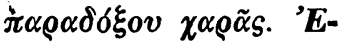

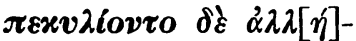

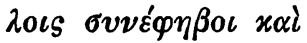
$20 \sigma v \nu \gamma v \mu \nu[\alpha] \sigma \tau \alpha i, X \alpha \iota-$

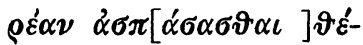

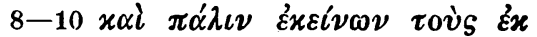

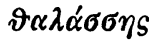

$12 \sigma v \nu \varepsilon v \chi \alpha i \pi v x \nu \alpha i \pi \alpha \rho ' \alpha \dot{\alpha} \mu 0_{-}$ $\tau \varepsilon \dot{\rho} \omega \nu$

13/14 $\mu \varepsilon \tau \alpha \xi \dot{v} \varphi \varepsilon \rho o ́ \mu \varepsilon \nu 0 s$ fehlt.

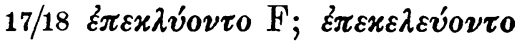
nach Reiske auch Hercher. 
Codex Thebanus.

$\lambda o \nu \tau \varepsilon \varsigma,[K \alpha \lambda \lambda \iota \varrho o ́ \eta \nu]$

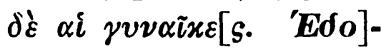

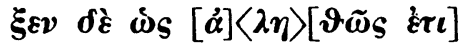

$25 x \alpha \lambda \lambda \varepsilon i \omega v[\ldots \ldots \ldots]$

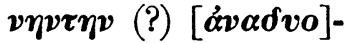

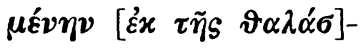

$\sigma \eta s . \quad \Pi \rho \circ[\varsigma \varepsilon \lambda \vartheta \dot{\omega} \nu \quad \delta \dot{\varepsilon}]$
Codex Florentinus.

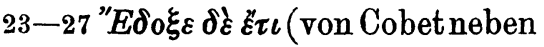
$\varepsilon \alpha v \tau \tilde{\eta}$ s gestrichen) $x \alpha i(x \alpha \lambda$ $\lambda i \omega v$ Reiske) $\alpha \dot{v} \tau \alpha \tilde{i} S$ ( $\dot{\varepsilon} \alpha v \tau \tilde{\eta} S$

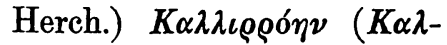

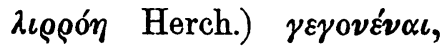

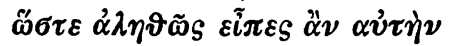

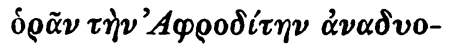
$\mu \varepsilon \dot{v} \eta \nu$

\section{Kolumne VII.}

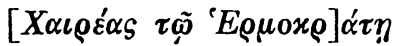
$\left[\begin{array}{lll}x \alpha i & \tau \tilde{\omega} & \pi \alpha \tau\end{array}\right] \rho i, \Pi \Pi \alpha \rho \alpha \lambda \alpha^{\prime}$ $[\beta] \varepsilon \tau \alpha \iota^{\prime \prime} \varphi \eta \sigma \iota v,, \tau \dot{\nu} \nu \pi \lambda o \tilde{v}-$

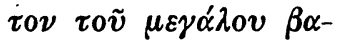

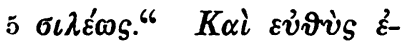

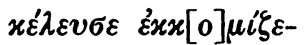

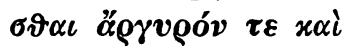

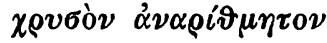

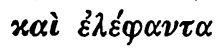

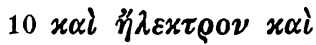
$\dot{\varepsilon} \sigma \vartheta \tilde{\eta} \tau \alpha \quad x \alpha \lambda \quad \pi \tilde{\alpha} \sigma \alpha \nu$

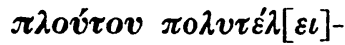

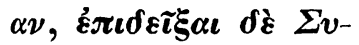

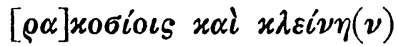

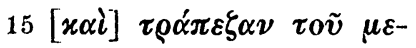
$[\gamma \alpha \dot{\alpha}] \lambda o v \quad \beta \alpha[\sigma \iota] \lambda \varepsilon^{\prime} \omega s \quad x \alpha \imath$ $[\varepsilon]$ v่vov́xovs xai $\pi \alpha \lambda$ -

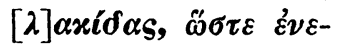
$[\pi \lambda \dot{\eta}] \sigma \vartheta \eta \pi \tilde{\alpha} \sigma \alpha \dot{\eta} \pi o^{-}$

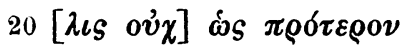

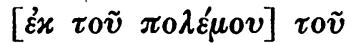

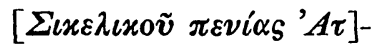

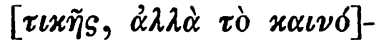

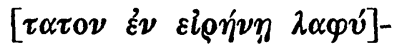

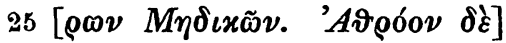

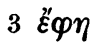

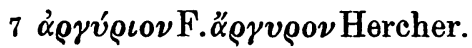
$\tau \varepsilon$ fehlt.

$9 \operatorname{\varepsilon i\tau } \alpha$

$12 \tilde{v} \lambda \eta s \tau \varepsilon_{\chi}^{\prime} \nu \eta s \tau \varepsilon$

$13 \varepsilon^{\prime} \pi \varepsilon^{\prime} \delta \varepsilon \iota \xi \varepsilon$. Es fehlt $\delta \varepsilon^{\prime}$.

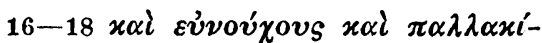
$\delta \alpha_{S}$ fehlt.

VI. 24 meine Kopie hat $\xi \varepsilon \nu \delta \varepsilon \omega \sigma . y .[\ldots \ldots]$. Das zweite $N$ mag ich aus dem Anfang von $\Lambda H$ verlesen haben. Vgl, unten S. $246 \mathrm{f}$. 
Codex Thebanus.

[ $\tau \dot{o} \pi \lambda \tilde{\eta} \vartheta 0 S \alpha \dot{\alpha} \nu \varepsilon \beta \delta \eta \sigma \varepsilon v]$

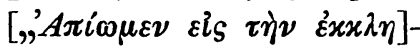

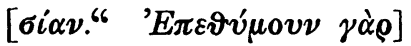

Codex Florentinus.

$27 \stackrel{\alpha}{\xi} \iota 0 \tilde{v} \mu \varepsilon \nu \mathrm{F} ; \dot{\alpha} \pi i \omega \mu \varepsilon \nu$ Beck.

\section{Kolumne VIII.}

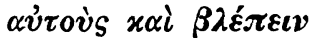

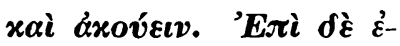

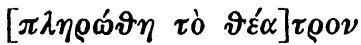

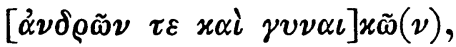

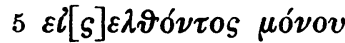

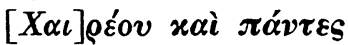

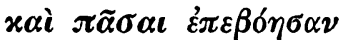

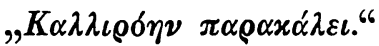

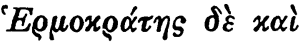

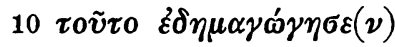

$\varepsilon \imath_{\zeta} \alpha \gamma \alpha \gamma \dot{\omega} \nu \tau \eta \dot{\nu} \nu \vartheta v \gamma \alpha-$

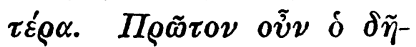

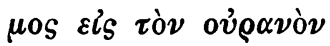

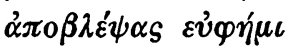

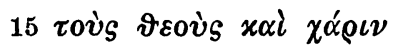

$\eta \dot{\eta} \pi i \sigma \tau \alpha \tau 0 \mu \tilde{\alpha} \lambda \lambda o \nu \dot{v}-$

$\pi \grave{\varepsilon} \rho \tau \tilde{\eta} S \quad \dot{\eta} \mu \varepsilon \alpha_{\alpha} \alpha \alpha v^{\prime}$

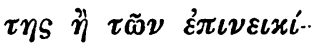

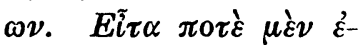

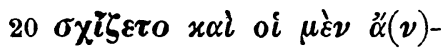

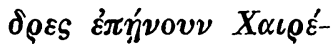

$\alpha \nu, \alpha i \delta \dot{\varepsilon} \gamma v \nu \alpha \tilde{i} x \varepsilon S \quad K \alpha \lambda-$

$\lambda \iota \varrho \delta ́ \eta \nu, \pi 0 \tau \dot{\varepsilon} \delta^{\prime} \alpha \tilde{v}^{\tilde{v}} \pi \alpha^{\prime}-$

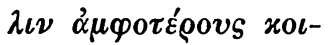

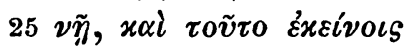

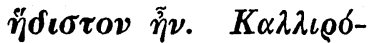

$\eta \nu \mu \dot{\varepsilon} \nu$ ஸे $\dot{\varepsilon} \varkappa \pi \lambda \sigma \tilde{v}$

$[\ldots \ldots \ldots \ldots \ldots]$

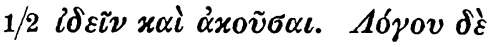

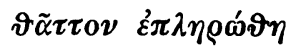

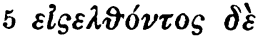

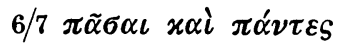

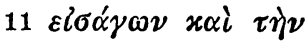

$14 \dot{\alpha} \pi 0 \beta \lambda \varepsilon \dot{\psi} \psi \alpha$ auch $\mathrm{F} ; \dot{\alpha} \nu \alpha \beta \lambda \varepsilon^{\prime}-$ $\psi \alpha s$ corr. Hercher.

$18 \tau \tilde{\eta} S \tau \tilde{\omega} \boldsymbol{\nu}$

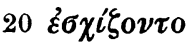

$23 \delta \tau \grave{\varepsilon}$

$26 \ddot{\eta} \delta \iota 0 \nu$

$27 \dot{\omega} s \grave{\alpha} \nu$

Die beiden Abschnitte, für die wir jetzt zwei Codices haben, gehören zu denjenigen Partien des Romans, in denen die Herausgeber an der Lesung des Florentinus nur wenig Anstofs genommen haben. Auch Cobet bringt in seinen Nachträgen zu Herchers Ausgabe (Mnemosyne VIII 229 ff.) für diese Abschnitte nur wenige Be- 
merkungen. ${ }^{1}$ ) Im Ganzen sind etwa ein Dutzend Verbesserungen zu dem überlieferten Text vorgeschlagen worden. ${ }^{2}$ ) Um so bemerkenswerter ist es, dafs der Codex Thebanus eine solche Fülle von Abweichungen bringt. Doch das Wichtigste sind nicht die einzelnen Verbesserungen, sondern der Einblick, der uns in die Textgeschichte eröffnet wird. Wenn ich recht gesehen habe, ergiebt die Vergleichung der beiden Handschriften, dals der Florentinus und der Thebanus zwei verschiedene Redaktionen des ursprünglichen, uns nicht bekannten Urtextes des Chariton darstellen.

Ich greife zunächst die beiden Stellen heraus, die die stärksten Abweichungen aufweisen.

Die eine ist III 9-20. Dionysios hat den Brief der Geliebten empfangen, hat ihn geküfst, dann geöffnet und an die Brust gedrückt, als wäre es die Geliebte selbst. Der Florentinus fährt fort: $x \alpha i \quad \xi \pi i$

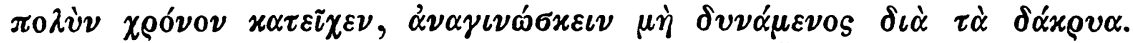

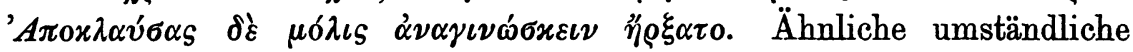
Schilderungen der Gemütsbewegungen sind passim in den griechischen Romanen zu finden. ${ }^{3}$ ) Bei der bekannten Vorliebe des Chariton für Wiederholungen kann die folgende Parallele als Stütze für die Gesamt-

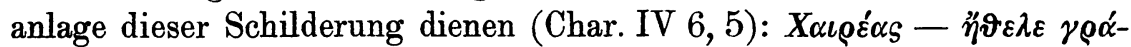

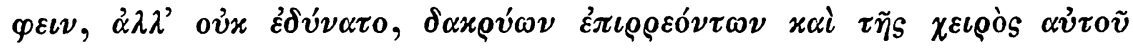

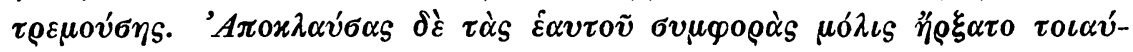

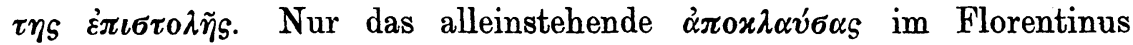

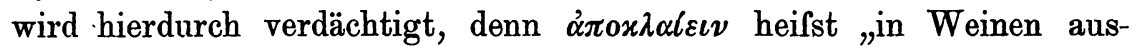
brechen" oder "beweinen". Ersteres ist hier wegen des vorhergehenden $\delta \iota \grave{\alpha} \tau \dot{\alpha} \delta \alpha ́ x \rho v \alpha$ ausgeschlossen. Für die zweite Bedeutung aber fehlt

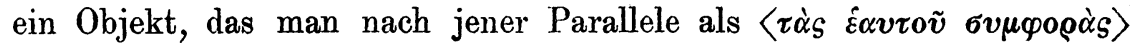
vielleicht um so lieber ergänzen möchte, als dieselbe Phrase auch in

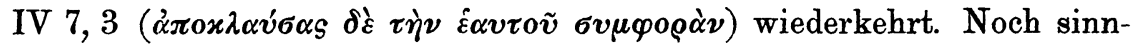
gemäfser wäre für diese Situation die Bedeutung ,sich ausweinen“, die freilich bisher nur für das Medium bezeugt ist; man müfste also $\dot{\alpha} \pi 0-$ $x \lambda \alpha v \sigma \alpha \dot{\alpha}\langle\mu \varepsilon \nu 0\rangle_{S}$ emendieren, wenn man nicht dort auch dem Aktivum eine durch die Lexica nicht bezeugte Bedeutung zutrauen will. Schlimm-

1) Vgl. auch Cobet, Variae lectiones ${ }^{2} 1873$ S. $169 \mathrm{ff}$.

2) Einige von Reiskes Emendationen (in der Editio princ. von D'Orville 1750) sind bei erneuter Revision in der Hs selbst gefunden worden, so $x \varepsilon v \grave{\eta}$ (oben

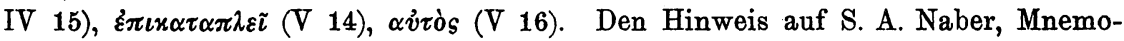
syne N. S. VI (1878) S. 190 ff. und F. W. Schmidt, J. Jahrbb. 125 (1882) S. 185 ff. verdanke ich Richard Förster.

3) So schon in dem ältesten Roman, den wir kennen, dem Ninosroman, A IV $26 \mathrm{ff}$ Vgl. Hermes XXVIII S. $172 \mathrm{f}$. 
sten Falls steckt also in dem $\dot{\alpha} \pi 0 x \lambda \alpha v ́ \sigma \alpha s$, an dem übrigens die Herausgeber bisher keinen Anstofs genommen haben, ein Fehler. Sonst ist diese Schilderung durchaus anschaulich und durch jene Parallele gestützt.

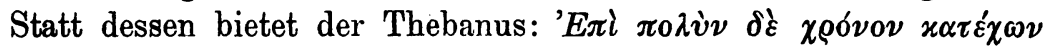

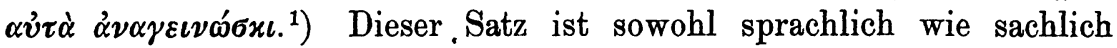

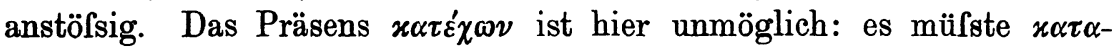
$\sigma \chi \grave{\omega} \nu$ heifsen. Doch das könnte immerhin ein Schreibfehler sein. Ferner ist $\alpha \dot{v} \tau \dot{\alpha}$ nicht gut, nachdem vorher von der $\dot{\varepsilon} \pi \iota \sigma \tau 0 \lambda \grave{\eta}$ und nicht mehr

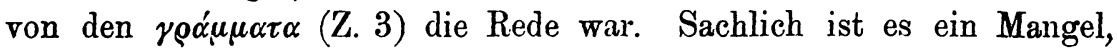
dafs nach dieser Version Dionysios beim Lesen des Briefes gar nicht in Thränen ausbricht, zumal der Dichter kurz vorher ausdrücklich gesagt hat, dafs Dionysios schnell vom König fortging, um weinen zu

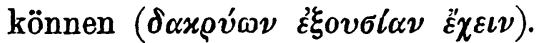

Während a priori bei derartigen Abweichungen die Möglichkeit der Interpolation ebenso wie die der Verkürzung zuzugeben ist, ist hier kein Zweifel, dafs der vollere Florentinus den ursprünglicheren Text bietet, während der Thebanus eine ungeschickte Verkürzung giebt.

Dagegen scheint mir das umgekehrte Verhältnis in den unmittelbar folgenden Sätzen vorzuliegen. Im Codex Thebanus ist das Lesen des Briefes in der Weise dargestellt, dafs die volle Adresse und der erste Satz - vgl. Char. VIII 4, 4/5 - wörtlich mitgeteilt werden, mit Hinzufügung der Ausrufe oder Liebkosungen, mit denen Dionysios das Lesen begleitet. In dieser Darstellung, für die ich auf S. 234 verweise, ist sprachlich alles in Ordnung bis auf einen Punkt. Wenn man ov่u $\varepsilon^{\prime} \chi \omega$ in Z. $15 / 6$ mit dem folgenden $\chi \alpha \iota \varrho \varepsilon \iota \nu$ verknüpft, so würde derselbe Gedanke mit $\pi \tilde{\omega} s \delta v ́ v \alpha \mu \alpha \iota$ nochmals zum Ausdruck kommen, und andrerseits würde $\tau \tilde{\omega} \tilde{\alpha} \nu \delta \varrho i$ eine Verschlechterung gegen-

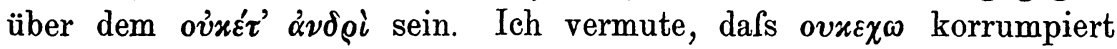

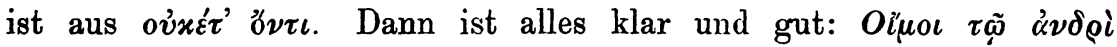

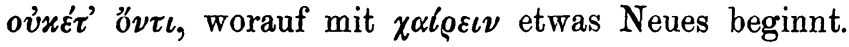

1) Nur nebenbei sei auf die orthographischen Eigentümlichkeiten des Thebanus hingewiesen, die in der Schreibart jener Zeit, in der der Codex entstand, begründet sind und weder mit Chariton noch mit der Redaktion etwas zu schaffen haben. Dahin gehören die damals üblichen Vertauschungen von $\iota$ für $\varepsilon \iota$ ( $\pi \alpha \iota \delta i \alpha \nu$

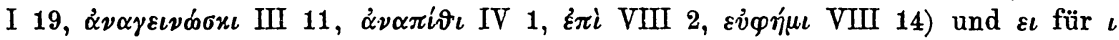

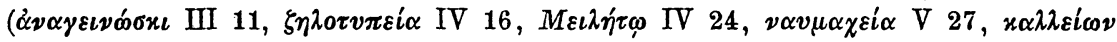

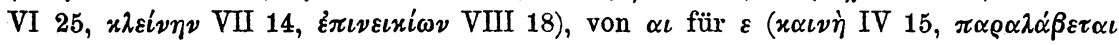

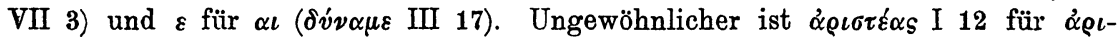

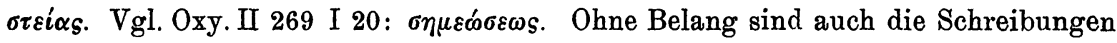

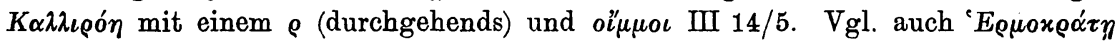

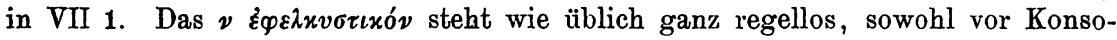
nanten (I 2, 13, VI 24) wie vor Vokalen (passim), auch fehlt es vor Vokalen (VII 5). 
Dagegen thut der Florentinus die Verlesung der Briefadresse mit

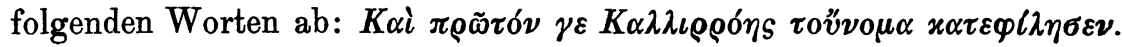

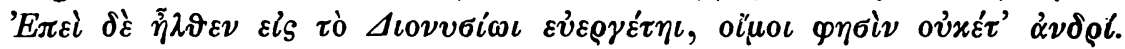
Eine wesentliche Kürzung! Die Spielerei mit dem $\chi \alpha\lfloor\varrho \varepsilon \iota \nu$ der Adresse ist völlig übergangen. Die Darstellung ist aber auch ungeschickt, denn

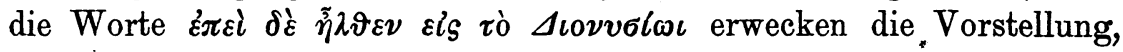

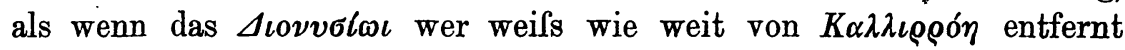
wäre, während es doch unmittelbar folgte. Dieser Darstellung liegt also keine anschauliche Vorstellung von der Adresse zugrunde.

Auch hier können wir nicht schwanken, wer die ursprüngliche Lesart hat: dieses Mal ist es der Thebanus, dem wir jetzt zum ersten Mal die Kenntnis des volleren Wortlautes verdanken, während der Florentinus eine stark, und zwar ungeschickt verkürzende Bearbeitung bietet.

In beiden Fällen hat sich die vollere Form als die ursprüngliche erwiesen. Für die Annahme einer erweiternden Bearbeitung eines ursprünglich kürzeren Textes hat sich kein Argument gefunden.

Ein ähnlicher Unterschied in der Ausführlichkeit zeigt sich Kol. IV 3-10. Der Florentinus erzählt die Begrüfsung des Dionysios mit dem Knaben - bis zu den Worten des Vaters - folgendermalsen: $\Theta \varepsilon \alpha \sigma \alpha \dot{\alpha} \mu \varepsilon v o s$ - wofür Hercher unter Hinweis auf den Homer-

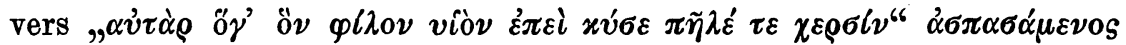

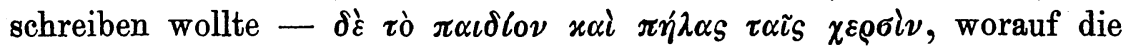
Ansprache folgt. Es ist von den Herausgebern nicht bemerkt worden, dals dieser Satz den durch die vorangegangene Erzählung gegebenen Voraussetzungen nicht gerecht wird. Dionysios hat sich vorher, um

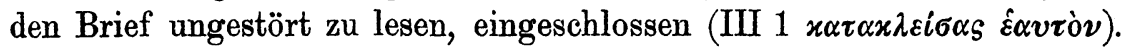
Wie soll er jetzt den Knaben, selbst angenommen, dafs er ihn von seiner

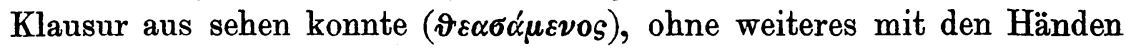
streicheln können? Mufste da nicht vorher erzählt sein, wie sie zu einander gekommen sind? Und dieses Desideratum wird in der That durch den volleren Text unseres Thebanus befriedigt, der sich so durch

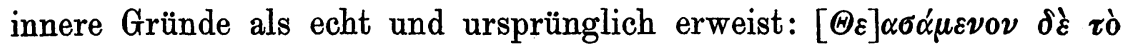

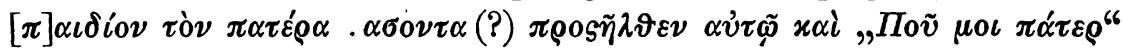

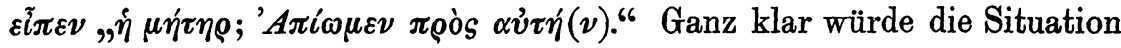
erst, wenn das Participium . $\alpha \sigma o \nu \tau \alpha$ mit Sicherheit ergänzt würde. Vielleicht bezeichnete dies das Verlassen der Klausur. Meine Lesung kann kaum richtig sein; ich wüfste wenigstens keine Ergänzung zu . $\alpha \sigma o \nu \tau \alpha$. Angenommen, dafs ich $\alpha$ mit $\lambda$ und $\sigma$ mit $\vartheta$ verwechselt habe, was bei dieser Unciale nahe liegt und bei einem Palimpsest wohl entschuld-

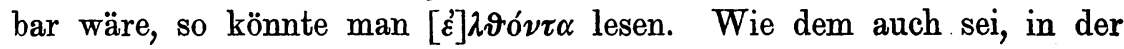


Hauptsache ist der Vorgang klar: Der Knabe läuft auf seinen Vater, als er ihn kommen sieht, zu und fragt ihn: „Wo ist die Mutter? Wir wollen zu ihr gehen." 1 )

Das alles hat der Redaktor des Florentinus, denn von einem solchen dürfen wir wohl schon reden, übergangen. Wie ist nun seine Verkürzung zu erklären? Aus dem angeführten Passus des Thebanus

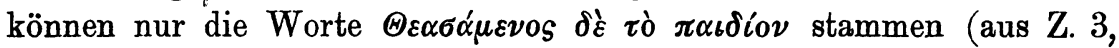
mit Veränderung der Konstruktion). Woher hat er die Homerremi-

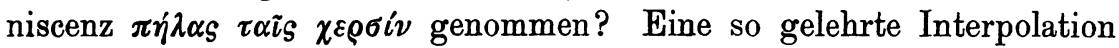
wird man dem Redaktor, der dazu noch kürzen wollte, gewils nicht zutrauen. Das ist sicher Charitonisch. Und damit ist uns der Weg für die Erklärung des Thebanus gewiesen: das Homerzitat mufs in dem Urtext zwischen den Worten des Knaben und der Antwort des Vaters gestanden haben. Diese Annahme ist um so einleuchtender, als es ungewöhnlich wäre, wenn wie jetzt im Thebanus die Worte des Vaters $\sigma \dot{v} \mu \grave{\varepsilon} \nu \dot{\alpha} \pi \varepsilon \dot{\lambda} \lambda \varepsilon v \sigma \alpha \iota \iota \tau \lambda$. unmittelbar, ohne erzählende Einleitung auf die Kindesworte folgten. Dafs also zwischen IV 8 und 9 eine Lücke an-

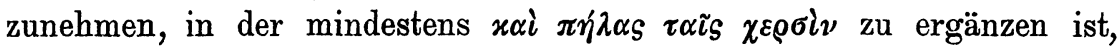
scheint mir sicher, und da $\pi \eta^{\prime} \lambda \alpha s$ nicht ohne Beziehung auf ein Objekt stehen wird, so wird auch $\tau$ ò $\pi \alpha \iota \delta i o \nu$ vorhergegangen sein. Es fehlt uns nur noch das passénde Partizipium zu diesem. Man könnte viel-

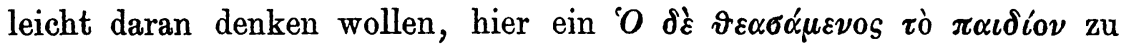
ergänzen, weil dann die kürzere Fassung des Florentinus nicht notwendig eine tiefgreifende redaktionelle Änderung zu sein brauchte, sondern einfach ein Schreibfehler sein könnte, indem wegen der Ähnlichkeit der Satzanfänge die erstere Periode überschlagen wäre. Aber abgesehen von der Kakophonie, würde $\vartheta \varepsilon \alpha \sigma \alpha ́ \mu \varepsilon v o s$ an der zweiten Stelle nicht an seinem Platze sein. Denn nachdem der Knabe den Vater angeredet hat, wird der Dichter nicht sagen, dafs der Vater ihn "erblickt" oder auch „anblickt". Wenn hier schon einmal erraten werden soll, was der Dichter geschrieben haben könne, so halte ich mich an den Homervers, auf den Hercher hingewiesen, und schlage der Sprach-

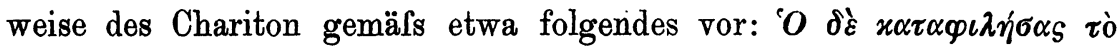

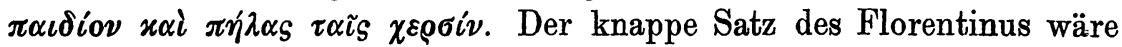
dann zusammengeflickt aus dem leicht veränderten $\vartheta \varepsilon \alpha \sigma \alpha ́ \mu \varepsilon \nu o \nu ~ \delta \grave{\varepsilon}$ tò $\pi \alpha \iota \delta i o v$ in Z. 3 und dem in der Lücke zwischen 8 und 9 anzu-

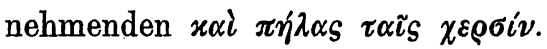

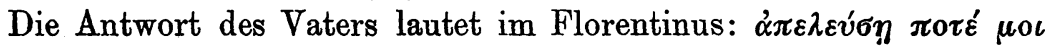

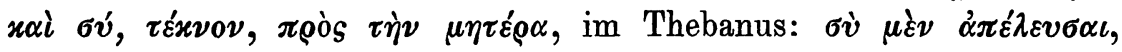

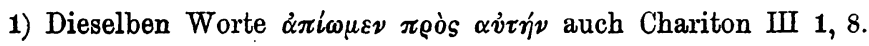




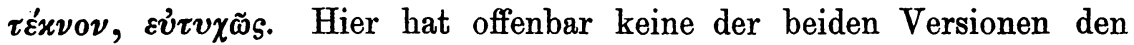
ursprünglichen Wortlaut. Nicht nur, dafs $\varepsilon v \dot{\tau} v \chi \tilde{\omega} s$ im Florentinus fehlt, auch das xai vor $\sigma \dot{v}$, das einen hier ganz unpassenden ${ }^{1}$ ) Hinweis auf die Mutter enthält, weist auf eine Verderbnis des Textes hin. Kai steht

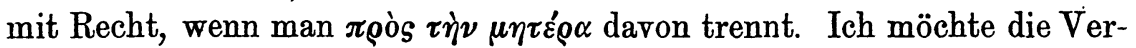
mutung wagen, dals das Original etwa folgendermalsen lautete: $\dot{\alpha} \pi \varepsilon \lambda \varepsilon v \dot{\sigma} \eta$

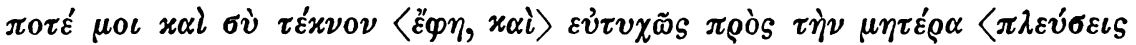
resp. $\pi \lambda \varepsilon v ́ \sigma \eta$ oder Optativ? $\rangle$, oder welches Verbum man hier wählen will.

Die Analyse dieser beiden Hauptstellen, an denen die durchgreifendsten Abweichungen vorliegen, hat ergeben, dafs weder der Florentinus noch der Thebanus den Urtext des Chariton überliefern, sondern zwei verschiedene, völlig unabhängig von einander gearbeitete Redaktionen des Charitonischen Textes darstellen, denen beiden die Tendenz innewohnt, den Urtext zu verkürzen. Wir haben damit eine Basis gewonnen für die Beurteilung der anderen Stellen, an denen geringfügigere oder aber an sich schwerer zu erklärende Abweichungen vorliegen. Betrachten wir diese der Reihe nach, zunächst soweit sie Spuren redaktioneller Bearbeitungen enthalten.

Ob II 6-9 hierher gehört, ist fraglich. In dem Text, wie ich ihn oben vermutungsweise nach $F$ hergestellt habe, ist das $x \alpha i$ vor $K \alpha \lambda \lambda \iota \varrho o ́ \eta v$ in Z. 8 jedenfalls unrichtig, denn etwas anderes als Kallirrhoë hätte der König ihm gar nicht „zurückgeben“ können. Es fragt sich nur, ob das eine unrichtige Zuthat eines Schreibers oder vielleicht ein Residuum aus einer volleren Gestalt des Satzes ist, aus der dann die Worte des Florentinus durch Verkürzung entstanden wären. Ich bemerke, dafs der in F überlieferte Text für Z. 7 etwas zu kurz er-

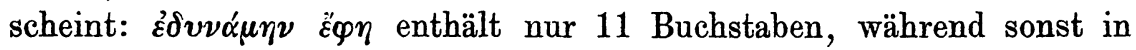
dieser Kolumne meist 14-16 stehen. Freilich wäre ein solcher Wechsel nicht ganz ausgeschlossen (vgl. VII 9, auch hier II 28). - Auch ob in Z. 1-6 der in F überlieferte Text wie oben unverändert einzusetzen ist, kann fraglich erscheinen: es ergeben sich dadurch einmal 16, zweimal 17 und zweimal 18 Buchstaben. Das ist für die Gewohnheit dieser Kolumne etwas reichlich. So halte ich es nicht für ausgeschlossen, dafs hier Abweichungen des Textes vorgelegen haben, deren Kenntnis sich uns jetzt entzieht.

Verschiedenheit der Konstruktionen liegt in II 15-18 vor. Die Mitteilung des Königs, dals Dionysios als erster Wohlthäter des königlichen Hauses geführt werden solle, ist in F als Hauptsatz seiner Ernennung zum

1) Das $x \alpha \imath$ ist von den Herausgebern bisher nicht beanstandet worden. Da aufser dem Knaben niemand zur Mutter gehen wird, ist die jetzige Überlieferung jedenfalls verderbt. 
Satrapen von Jonien angegliedert, während in Th die beiden Gnadenakte gleichmälsig von $\delta i \delta \omega \mu \iota$ abhängig gemacht sind. Da die letztere Konstruktion die kunstvollere ist, wird sie die ursprüngliche sein.

In $\mathrm{F}$ ist aber nicht nur die Konstruktion geändert, sondern es ist

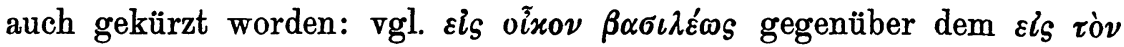

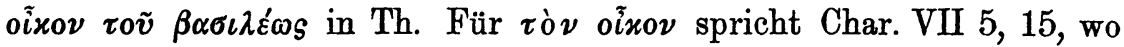
dieser selbe Gnadenakt angekündigt wird mit den Worten: $\alpha \nu \alpha \gamma \rho \alpha \dot{\psi} \omega$

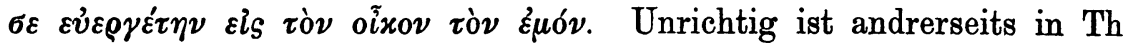
der Artikel vor $\beta \alpha \sigma \iota \lambda \varepsilon \varepsilon^{\omega} \omega s$, da Chariton sonst, wie üblich, den Perserkönig $\beta \alpha \sigma \iota \lambda \varepsilon v_{S}$ zu nennen pflegt. Da er ihn aber gelegentlich auch $\delta \mu \varepsilon^{\prime} \gamma \alpha_{S} \beta \alpha \sigma \iota \lambda \varepsilon v_{S}^{\prime}$ nennt (vgl. Kol. VII 4/5), so ist es mir nicht unwahrscheinlich, dafs $\tau$ võ $\beta \alpha \sigma \iota \lambda \varepsilon \dot{\omega} \omega s$ hier eine fehlerhafte Verkürzung aus $\tau о \tilde{v} \mu \varepsilon \gamma \alpha \dot{\lambda} \lambda o v \beta \alpha \sigma \iota \lambda \varepsilon \dot{\varepsilon} \omega s$ ist. Diese Ausdrucksweise würde an dieser Stelle etwas Feierliches haben, was für die Situation gut passen würde. Vgl.

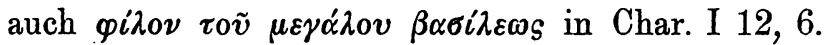

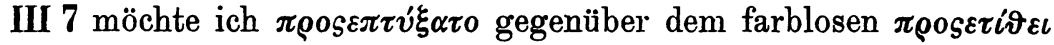
halten. Die Dürftigkeit des $\pi \rho \circ \varsigma \tau \iota \vartheta \varepsilon ́ v \alpha \iota$ wird erst klar, wenn man an

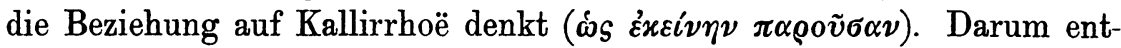

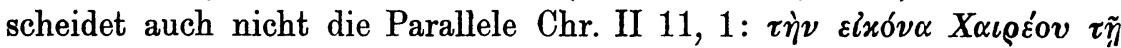

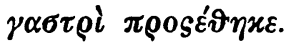

Schwierig ist die Beurteilung der Abweichungen in III 21-28. Was

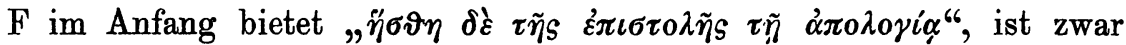
sprachlich unanstöIsig, aber sachlich ist bedenklich, dafs dieser Brief,

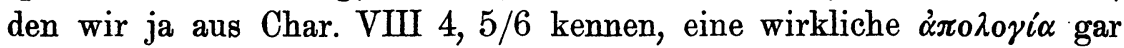
nicht enthält. Mir scheint der Grundgedanke dieses ganzen Passus vielmehr folgender zu sein: „Dionysios liest aus dem Brief eine Rechtfertigung der Geliebten heraus; er glaubt zwischen den Zeilen zu lesen, dafs sie ihn gezwungen verlassen habe, denn Eros macht die Liebenden leichtgläubig." Vielleicht gelingt es, durch Ineinanderschiebung der beiden Redaktionen dem ursprünglichen Text näher zu kommen. Der

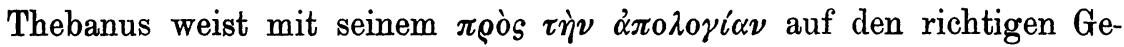
danken hin. Es fragt sich nur, welches Verbum an der Spitze des Satzes, wo ich $\eta \sigma . \varepsilon \tau o$ las, herzustellen ist. Ist die Lesung richtig, so wüfste ich keine andere normale Ergänzung als $\eta^{\prime} \sigma[\vartheta] \varepsilon \tau o$, was nicht befriedigt.

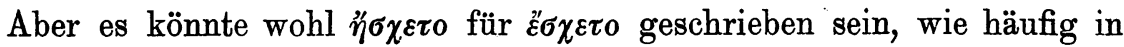
vulgären Texten, und dies würde einen guten Sinn ergeben: „er hielt sich an den Brief, klammerte sich an ihn, um sie zu rechtfertigen."

Soll nun der Redaktor des Florentinus das $\ddot{\eta} \delta \varepsilon \sigma \vartheta \alpha \iota$ sich selbst erdacht haben? Gewifs nicht, zumal der Begriff hier durchaus am Platze, ja notwendig ist, nachdem vorher nur von dem Wehklagen des Dionysios gesprochen ist. Man wird die beiden Redaktionen zu ver- 
binden haben. Vorausgesetzt, dafs Chariton etwa folgendermalsen ge-

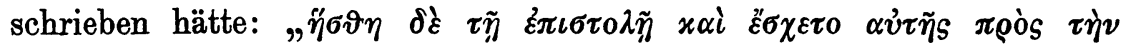

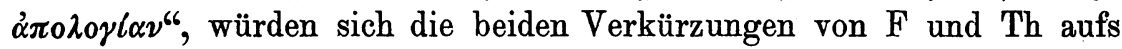
beste erklären.

Auch im folgenden Satz sind vielleicht die beiden Redaktionen in-

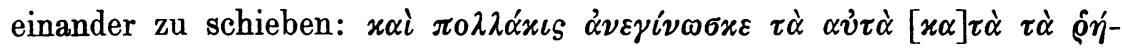
$\mu \alpha \tau \alpha$. Oder ist in Th $[\alpha \hat{v}] \tau \dot{\alpha}$ zu ergänzen? Dann würde wohl $\alpha \dot{v} \tau \dot{\alpha} \tau \dot{\alpha}$ $\hat{\varrho} \eta \dot{\mu} \alpha \tau \alpha$ verschrieben sein für $\tau \grave{\alpha} \alpha \dot{v} \tau \dot{\alpha} \varrho \hat{\eta} \mu \alpha \tau \alpha$.

Stärker gehen die Handschriften wieder im folgenden aus einander.

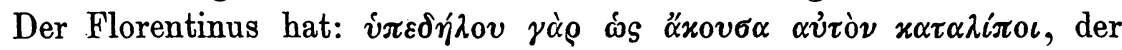

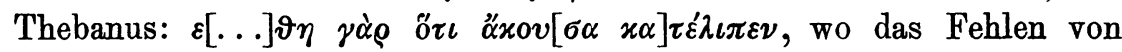
$\alpha \tilde{v} \tau o \tilde{v}$ nur Schreibfehler ist. Was F giebt, ist fein und zutreffend: die Irrealität der Vorstellung ist durch $\dot{\omega} s$ mit dem Optativ hervorgehoben. Auch wird mit Recht nur gesagt, dafs der Brief es „andeute“, dafs sie ihn gegen ihren Willen verlassen habe, denn ausdrücklich sagt er es nicht. Der Aorist Passivi in Th $\varepsilon[\ldots] \vartheta \eta$ mufs einen ganz anderen Gedanken als $\dot{v} \pi \varepsilon \delta \eta^{\prime} \lambda o v$ ausdrücken, da ö $\tau \iota$ mit dem Indikativ folgt. Wenn ich $\varepsilon[\pi i \sigma] \vartheta \eta \nu$ (für $\dot{\varepsilon} \pi \varepsilon i \sigma \vartheta \eta \nu$ ) vermute, so finde ich eine

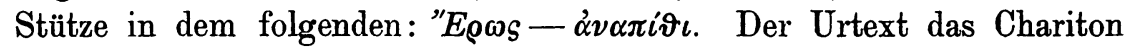
wird derartig gewesen sein, dafs sowohl die Lesungen von $\mathrm{F}$ wie von Th daraus entnommen werden konnten. Ich vermute etwa folgendes:

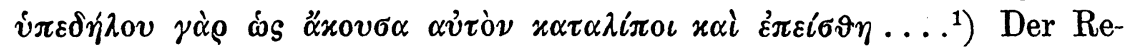
daktor von $\mathrm{F}$ würde also die Schlufspointe, dafs Dionysios es auch glaubte, ausgelassen haben, während der von Th auf das $\boldsymbol{v} \pi 0 \delta \eta \lambda o v v$

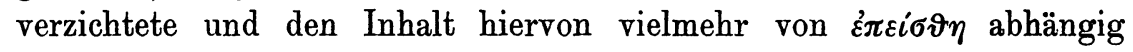
machte. ${ }^{2}$ )

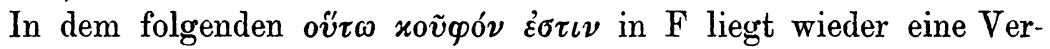
kürzung vor, wie die Lücke in Z. 28 zeigt. Das von mir ergänzte $\delta \dot{\eta}(?)$ $\varphi v ́ \sigma \varepsilon \iota$ würde echt Charitonisch sein. Vgl. die Beispiele bei Cobet, Mnemosyne VIII S. 254.

Nebenbei sei bemerkt, dafs den 4 Kolumnen, die zwischen IV und $\mathrm{V}$ fehlen, in $\mathrm{F}$ ungefähr ebenso viel Text entspricht wie den Kol. I-IV und V-VIII. Daraus folgt natürlich nichts für die Übereinstimmung des Inhaltes.

Der Anfang von Kol. V zeigt eine vollständige Diskrepanz gegenüber F. Was ich in Z. 1 und 2 gelesen habe, $\varphi \iota \lambda \alpha \varrho \gamma\left[v \rho .\right.$. und $\pi \varepsilon \varepsilon^{\prime} \eta \mu[$,

1) Hiermit soll nur der Gedankengang angegeben sein. Vielleicht folgte noch ein Satz mit ö $\iota \iota$ und dem Indikativ, der den Gedanken in anderen Worten wiederholte.

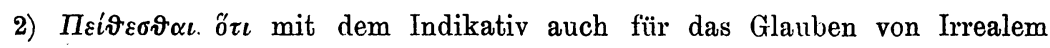
z. B. bei Chariton III 4, 10, VII 5,15 . 
kommt in $\mathrm{F}$ in dieser Gegend überhaupt nicht vor, und es ist schwer, irgend eine Beziehung zu dem Text von F zu finden. Der $\varphi \iota \lambda \alpha \dot{\rho} \gamma v[\rho \circ s$, der Geizige, liefse sich zwar mit dem Schatzfinder zusammenbringen,

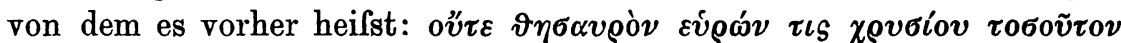

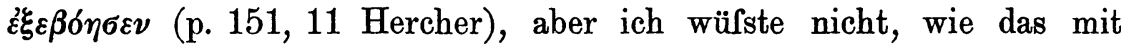

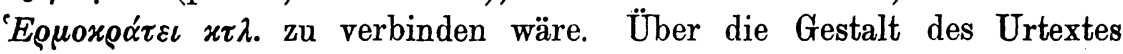
lärst sich hier keine Vermutung aufstellen. - Wenn in Th hinter

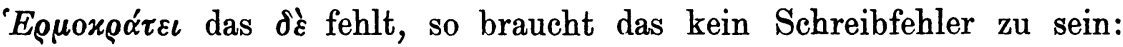
es kann hier in Th eine andere Satzkonstruktion gewesen sein.

Durchsichtig ist die Diskrepanz in V 21-23: hier ist klar, dafs

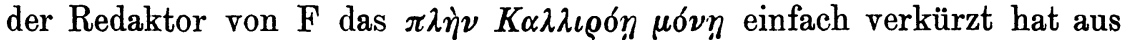

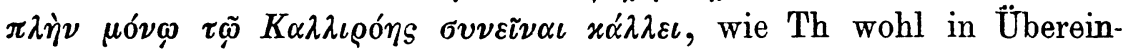
stimmung mit dem Urtext schreibt.

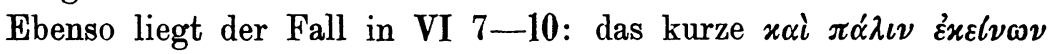

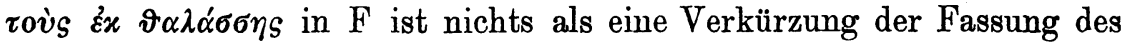
Thebanus, der auch hier gewifs den Urtext wiedergiebt: $\tau \tilde{\omega} \boldsymbol{\nu}] \delta \dot{\varepsilon} \alpha \dot{\alpha} \boldsymbol{x}$

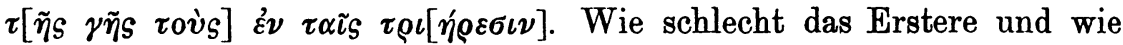
gut das Letztere ist, sieht man erst, wenn man die vorhergehende Parallele

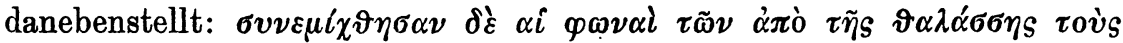

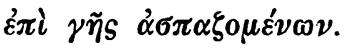

In den folgenden Worten VI 12 ist dagegen wieder Th kürzer: es

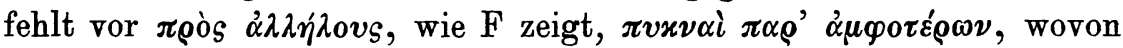

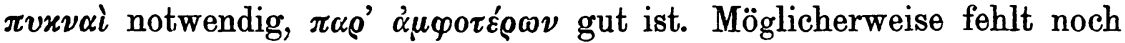
in beiden Codices ein Verbum finitum, aber nötig ist dies nicht. Vgl.

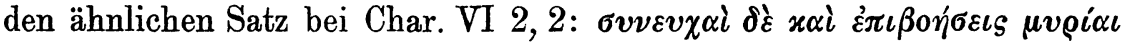

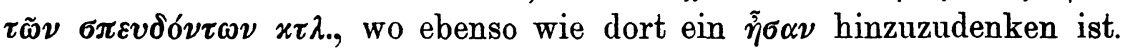
Diese Parallele bestärkt uns darin, in dem einfachen $\varepsilon \dot{v} \chi \alpha i$ in Th an Stelle von $\sigma v \nu \varepsilon v \chi \alpha i$ in $\mathrm{F}$ eine Verschlechterung zu sehen. ${ }^{1}$ )

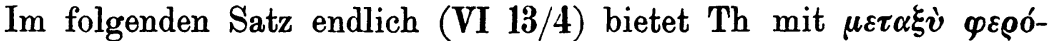
$\mu \varepsilon \nu$ os offenbar den Urtext, während in $\mathrm{F}$ wieder eine Kürzung vorliegt.

Über VI 23-28 kann ich nicht so bestimmt urteilen, da hier der Text von Th lückenhaft ist. Das $\dot{\omega} S[\tilde{\alpha}]\langle\lambda \eta\rangle[\vartheta \tilde{\omega} s$ in 24 ist nur eine nachträgliche Vermutung für die Lesung $\omega \sigma . v .[\ldots$ (s. oben S. 237). Ich glaube freilich, zumal $\dot{\alpha} \lambda \eta \vartheta \tilde{\omega} S$ im nächsten Satz auch in F vorkommt, dafs meine Lesung damit richtig korrigiert ist. Dagegen weifs ich mit $\nu \eta \nu \tau \eta \nu$ in 26 nichts anzufangen. Jedenfalls liegen hier starke

1) $\Sigma v v \varepsilon v \chi \alpha i$ empfiehlt sich vielleicht auch wegen des Hiatus. Vgl. Hercher, J. Jahrbb. 77 (1858) S. 165. Freilich nach dem neuen Einblick in die Bedeutung des Florentinus müssen wir uns gestehen, über Charitons Verhältnis zum Hiatus ein sicheres Urteil nicht fällen zu können. Vielleicht ist auch Char. III 5, 3 ó $\boldsymbol{o}_{0} \tilde{v}$ $\sigma v v \varepsilon v \chi \alpha i \mathrm{zu}$ schreiben. 
Kürzungen gegenüber $\mathrm{F}$ vor. Angenommen, Th habe als Hauptsatz nur

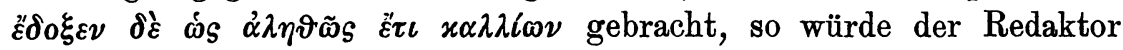

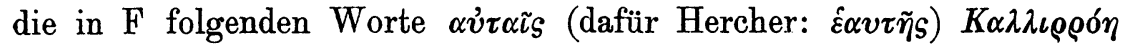

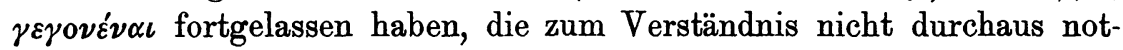

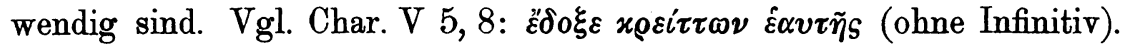
Übrigens halte ich Herchers Vorschlag $\varepsilon \alpha v \tau \tilde{\eta} \varsigma^{1}$ ) nicht für nötig. Vgl.

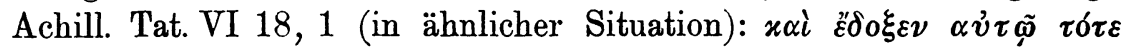

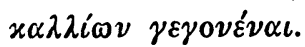

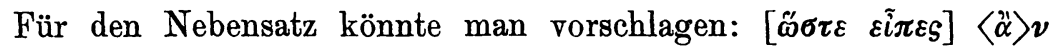

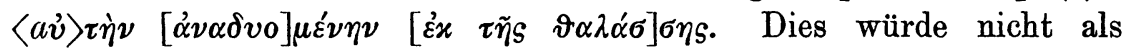
künstliche Verkürzung, sondern als Schreibfehler aufzufassen sein, da der Schreiber das $\tau \eta \nu$ in 'A

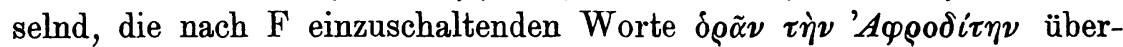
sehen haben müfste. Doch über Mutmafsungen komme ich nicht hinaus. Es ist nicht ausgeschlossen, dals Th eine ganz andere Konstruktion als $\mathrm{F}$ geschaffen hat. $K \alpha \lambda \lambda i \omega \nu$ ist übrigens eine schöne Bestätigung für Reiske, der so statt xai (F) emendierte.

Verschiedene Satzkonstruktionen liegen ferner in VII 5-18 vor

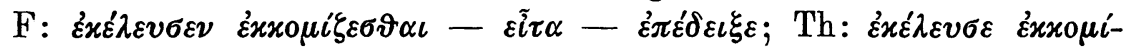
$\xi \varepsilon \sigma \vartheta \alpha \iota-\dot{\varepsilon} \pi \iota \delta \varepsilon \tau \xi \alpha \iota \delta \dot{\varepsilon}$. Vgl. hierzu II 15 ff. Ebenso wie dort glaube ich, dafs auch hier der Thebanus mit seiner strafferen Konstruktion das Richtige und Ursprüngliche giebt. Man beachte ferner, wie in $\mathrm{F}$ die Akkusative $x \alpha i$ x $\lambda i \nu \eta \nu ~ x \tau \lambda$. nachhinken, während sie in Th in einen wohl berechneten Gegensatz zu den vorhergehenden gestellt sind: Silber, Gold, Elfenbein, Elektron, Kleider und die ganzen Kostbarkeiten lälst Chaereas ausladen; besonders zeigen aber läfst er den Syrakusanern das Bett und den Tisch des Grofskönigs und seine (von F ganz übergangenen) Eunuchen und Kebsweiber. ${ }^{2}$ ) Es ist wohl kein Zweifel, welche Darstellung die ursprüngliche ist.

Zum Text dieses Abschnittes bemerke ich noch im Einzelnen: å cher auf Grund vieler Parallelen in đogvoov geändert hatte. Das folgende $\tau \varepsilon$ (Z. 7) ist gleichfalls gut, von F ausgelassen. Dagegen wird man das $x \alpha i$ in 9 für eine Verschlechterung halten müssen (vielleicht Schreibfehler). Gerade dies cĩ $\tau$, das der Redaktor von F im Chariton vorfand, hat ihn zu der unrichtigen Verteilung der Akkusative verführt. In 12 steht $\pi \lambda o v ́ \tau o v$ statt $\tilde{v} \lambda \eta s \quad \tau \dot{\varepsilon} \chi v \eta s \quad \tau \varepsilon$ (F). Man könnte die erstere Lesung durch Char. VI 9,6 stützen wollen, wo in ähn-

1) Vgl. dazu Cobet, Mnemosyne VIII a. a. O.

2) Sie begegnen auch in der ähnlichen Aufzählung Char. VI 9, 6. 


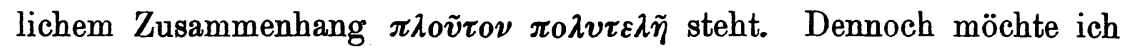
vँ$\lambda \eta s \tau^{\prime} \chi \nu \eta s ~ \tau \varepsilon$ für die Schreibung Charitons halten, gerade wegen ihrer Künstlichkeit und Gespreiztheit. Was wir bisher als Redaktorengriechisch nachgewiesen haben, war immer äufserst simpel und platt. Auch würde es gegen die Tendenz zu kürzen, die überall hervorgetreten ist, ver-

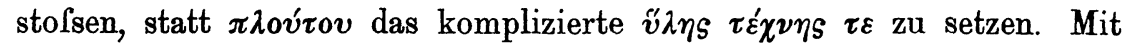
letzterem Ausdruck wird offenbar auf die beiden Gruppen hingewiesen, denen die vorhergenannten Einzelobjekte angehören: Silber, Gold, Elfenbein, Elektron gehören zur $v ́ \lambda \eta$, die Kleider zur $\tau \dot{\varepsilon} \chi v \eta$. Diese Spielerei ist echt Charitonisch. - Endlich ist noch die attische Schrei-

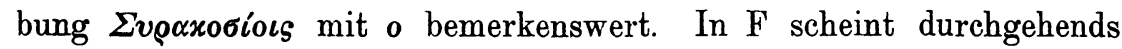
$\Sigma v \varrho \varrho \alpha x o v \sigma \iota s$ oder $\Sigma v \varrho \alpha x o v ́ \sigma \iota s$ zu stehen, wie D'Orville schrieb. Mit Recht tadelte es Hercher, dals Hirschig ohne weiteres überall die attische Form herstellte. Wenn er hinzufügte (J. Jahrbb. a. a. 0. 155) „bei einem Spätling wie Chariton durfte die Einführung jenes Atticismus nur auf Grund einer handschriftlichen Spur gewagt werden", so würden seine Bedenken durch das Zeugnis unserer alten Handschrift wohl jetzt geschwunden sein.

Endlich bringt uns auch die letzte Kolumne eine neue Satz-

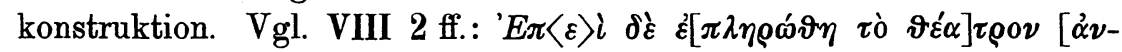

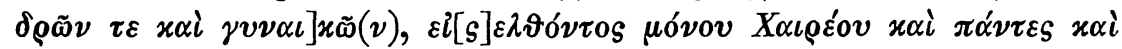

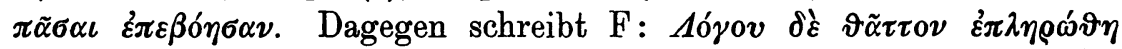

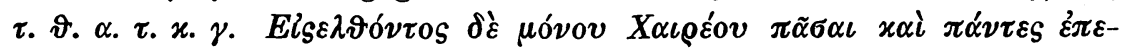

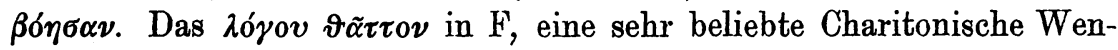
dung, ist sicherlich auch an dieser Stelle echt, denn der Dichter wird nicht versäumt haben, die grofse Schnelligkeit, mit der das Theater sich füllte, zu schildern. Vgl. die ganz ähnliche Darstellung Char. III

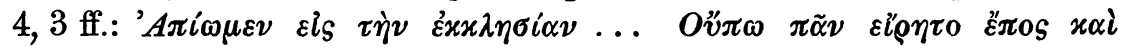

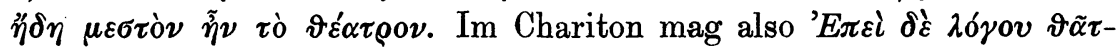

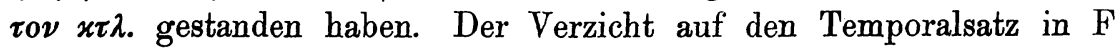
ist zwar nur eine sehr unbedeutende Kürzung, die sich kaum verlohnt, entspricht aber doch der mehrfach hervorgetretenen Abneigung dieses Redaktors gegen straffere Konstruktionen, resp. seiner Vorliebe für die Parataxe.

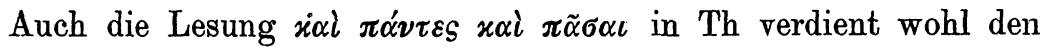
Vorzug, nur das erste xai könnte zweifelhaft sein. Die Umstellung

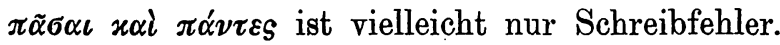

Damit wären wohl die Stellen, die sicher die Hand des Redaktors verraten, erschöpft. Es sei endlich noch auf diejenigen Abweichungen, soweit sie nicht schon oben ,zur Sprache kamen, hingewiesen, die sicher oder vermutlich als Schreibfehler zu betrachten sind. Freilich ist auch 
bei manchen von diesen noch die Möglichkeit einer beabsichtigten Änderung durch den Redaktor zuzugeben.

I) Im Thebanus.

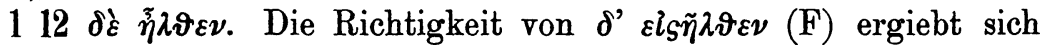

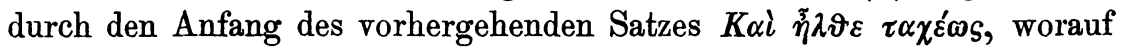
sein Zustand geschildert wird. Nur $\varepsilon i s \tilde{\eta} \lambda \vartheta \varepsilon v$ ergiebt einen Fortschritt der Handlung.

I 24 der Konjunktiv $\tau \alpha \varrho \alpha \chi \vartheta \tilde{\eta}$, sicher Schreibfehler für $\tau \alpha \rho \alpha \chi \vartheta \varepsilon i \eta$, wie der Florentinus schreibt, dem aber seinerseits wieder das $\varepsilon l$ fehlt. Das $\varepsilon l$ in Th beseitigt einen Anstofs, den Cobet durch den Vorschlag

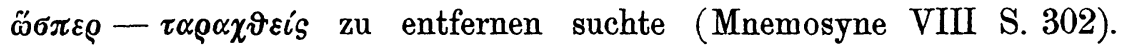
Durch Kombination der beiden Handschriften gewinnen wir jetzt die richtige Lesung:

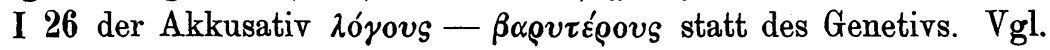
Char. IV 6,$2 ; \mathrm{V} 5,3 ; \mathrm{V} 7,1$.

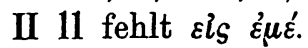

II 19 fehlt $\delta$.

III 6 ist xai statt kĩ $\alpha$ fehlerhaft. Man könnte freilich in dem ab-

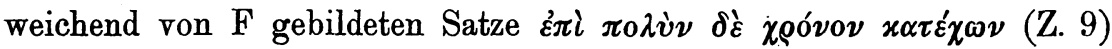
den dem $\pi \rho \tilde{\omega} \tau o \nu$ (Z. 4) entsprechenden zweiten Teil erkennen wollen. Aber würde dann nicht $\pi \varrho \tilde{\tau} \tau o \nu \mu \grave{\nu} \nu$ gesagt sein? Jetzt korrespondieren

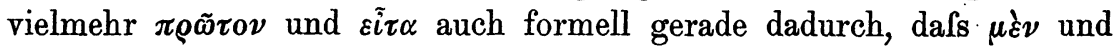
$\delta \dot{\varepsilon}$ bei ihnen fehlen.

III 20 dürfte dem $\sigma o v$ das $\sigma o \iota$ in $F$ vorzuziehen sein, denn nur dadurch kommt der Begriff der Wohlthätigkeit zum Ausdruck.

IV $19 / 20$ ist $\mu \varepsilon(\nu) \vartheta \alpha \gamma \alpha \nu$ völlig sinnlos. Hier liegt eine stärkere Korruptel unseres Thebanus vor, denn es fehlt nach F statt dessen:

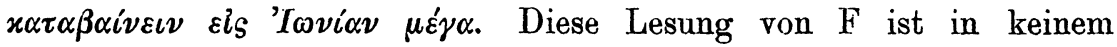
Punkte verdächtig. Wie die Korruptel in Th entstanden, ist schwer zu sagen. Man sieht auch nicht, was der Schreiber sich dabei gedacht haben könnte. Dies ist insofern nicht unwichtig, als es zeigt, dafs der Schreiber von Th - jedenfalls an dieser Stelle - keine Lust zum Erklären und Interpolieren gehabt hat. Er bietet etwas, das auch für ihn völlig sinnlos gewesen sein mufs. - Das vor-

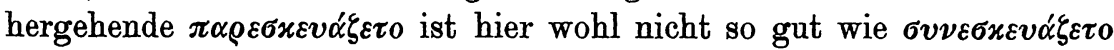
in $\mathrm{F}$, das speziell "sich reisefertig machen" bedeuten kann. Vgl. Char.

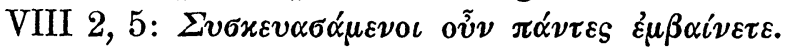

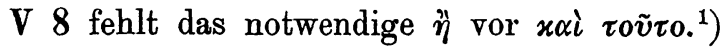

1) Dagegen möchte ich dies $x \alpha i$, das in Th wie in $\mathrm{F}$ steht, nicht mit Hercher

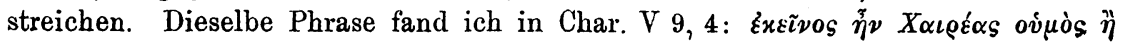


V 10 fehlt $\nu \tilde{v} \nu$ vor $\dot{\alpha} \lambda \eta \vartheta \tilde{\vartheta}$ s. Dagegen ist vorher $\varepsilon \tilde{i} \pi \varepsilon \nu$ in 9 wohl als Dittographie (vgl. 7) zu streichen.

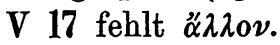

$\mathrm{V} 19$ ist das $\mu \eta x \varepsilon \varepsilon^{\tau} \iota$ in $\mathrm{F}$ wohl dem $\mu \grave{\eta}$ vorzuziehen.

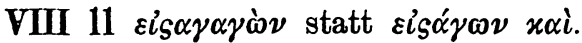

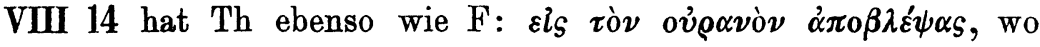

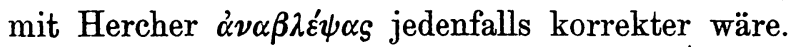

VIII 18 fehlt $\tau \tilde{\eta} s$ hinter $\ddot{\eta}$. Vgl. Char. I 1, 13.

VIII 26 ist $\ddot{\eta} \delta \iota \sigma \tau o \nu$ wohl schlechter als $\ddot{\eta} \delta \iota \nu \nu(F)$.

II) Im Florentinus.

I 14 fehlt $\dot{\xi} \xi \dot{\alpha} \varrho \chi \tilde{\eta} s$, vielleicht vom Redaktor ausgelassen.

I $16 \quad \delta \dot{\varepsilon}$ statt $\delta \grave{\eta}(\mathrm{Th})$.

I 17 fehlt $\mu \alpha^{\prime} \lambda \iota \sigma \tau \alpha$. Dafs Dionysios die hier aufgeführten Charaktereigenschaften hatte, ist vorher schon mehrmals mit ganz ähnlichen Worten gesagt worden. Vgl. Char. II 4, 1 und V 5, 1. Aber in diesem

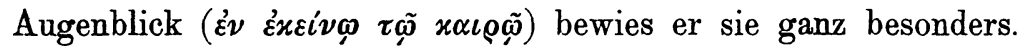

I 24 fehlt oǿ $\tau \omega s$, wie schon Hercher gesehen hatte, und jetzt durch Th bestätigt wird.

II $21 \varepsilon^{\prime} \chi \varepsilon \iota \nu$ hinter $\delta \mu \circ \lambda \circ \gamma \eta^{\prime} \sigma \alpha S$ schon von Hercher als Dittographie (vgl. Z. 24) mit Recht gestrichen, wie gleichfalls Th bestätigt. ${ }^{1}$ )

II $27 \delta i \delta \omega \sigma \iota \nu$ schlechter als $\dot{\varepsilon} \pi \iota \delta i \delta \omega \sigma \iota \nu$, das terminus technicus für das Überreichen von Briefen ist.

IV 22 fehlt $\pi 0 \lambda \lambda \tilde{\omega} \nu$.

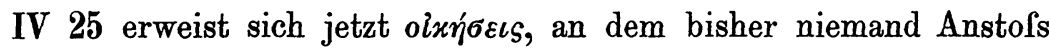
genommen hat, als ein eklatanter Fehler statt cixóvos. Wie schief war der Gedanke, dafs die Wohnungen (Plural!) der Kallirrhoë dem verlassenen Gatten ein Trost sein sollten! Nein, ihre Statuen, ihre Bilder, die er in Milet wiederfinden wird, die werden ihn trösten. Von einer goldenen Statue, die Dionysios selbst im Tempel der Aphrodite auf-

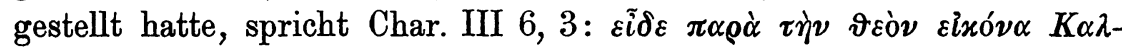

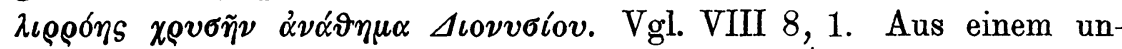
deutlich gewordenen kursiven $\varepsilon \iota^{x}$ konnte leicht o $\iota^{x}$ werden, das dann

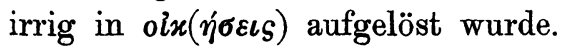

$\mathrm{V} 10$ fehlt $\zeta \tilde{\omega} \nu \tau \alpha$.

V $12 \varepsilon^{2} \chi \varepsilon \tilde{\tau} \tau$ statt $\varepsilon^{\prime} \xi \varepsilon \chi \varepsilon i \tau o$.

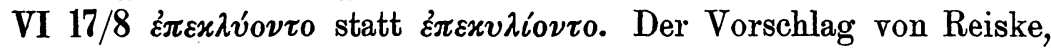

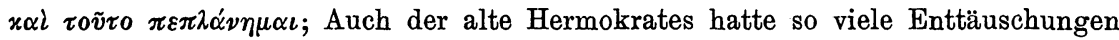
durchgemacht, dafs Chariton ihn mit besonderer Bitterkeit $x \alpha i$ voṽ sagen lassen konnte, auch wenn dem Leser die spezieile Beziehung unklar bleibt.

1) Zu $\chi \alpha \dot{\rho} \varrho \nu$ ó $\mu о \lambda o \gamma \varepsilon \tilde{\nu} \nu$ vgl. aufser Achill. Tat. III 10,6 und Longus I 26, 3 auch die Korrespondenzen des Fl. Abinnäus aus dem IV. Jahrh. in P. Lond. II S. 272 ff. 


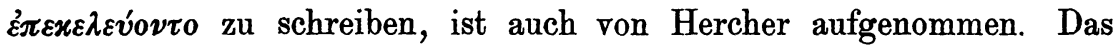
Richtige bringt unser Thebanus: die früheren Spielgenossen wälzten, drängten sich heran, um ihren Chaereas zu begrüfsen! Zu ध̇ $\pi \iota x v \lambda i \varepsilon \sigma \vartheta \alpha \iota$ vgl. das namentlich in den Ephesiaca des Xenophon so häufige $\pi \varrho 0-$

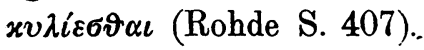

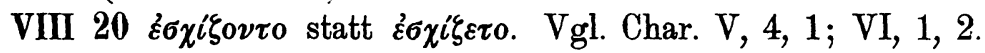

VIII $23 \delta \tau \grave{\varepsilon}$ statt $\pi 0 \tau \grave{\varepsilon}$. Schon Reiske hatte $\delta \tau \grave{\varepsilon}-\delta \tau \grave{\varepsilon}$ oder $\pi 0 \tau \grave{\varepsilon}$

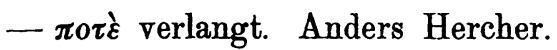

VIII $27 \stackrel{\alpha}{\nu}$ ist zu streichen, da es sich nicht um eine irreale Vorstellung handelt.

Fassen wir die obigen Beobachtungen zusammen. Es hat sich ergeben, dafs der Thebanus und der Florentinus zwei völlig unabhängig von einander durchgeführte Bearbeitungen des charitonischen Urtextes sind. Es hat sich ferner ergeben, dafs diese Bearbeitungen Kürzungen des Originals darstellen, denn in keinem der oben angefübrten Fälle ist eine Wahrscheinlichkeit dafür erwiesen worden, dafs etwa die kürzere Form die originale sei und die vollere eine spätere Erweiterung darstelle. Vielmehr haben überall die volleren Formen sich als sinngemärs, oft als notwendig und vielfach auch durch Parallelen als gut charitonisch erweisen lassen, während die kürzeren Formen sich deutlich durch ungeschickte Handhabung der Sprache, durch Schaffung sachlicher Lücken und andere Zeichen jüngerer Bearbeitung als nicht originell verrieten.

Das Mafs der Verkürzungen und überhaupt der Veränderungen können wir nur für die beiden im Thebanus erhaltenen Abschnitte konstatieren. Hier hat offenbar der Florentinus stärker gekürzt als der Thebanus. Dafs die beiden Redaktoren überall in diesem selben Verhältnis gekürzt haben, ist nicht nötig anzunehmen. Bürger (Hermes 27) hat gezeigt, wie der Epitomator der Ephesiaca des Xenophon sprungweise vorgegangen ist, bald einige Stellen in vollem Wortlaut hat stehen lassen, dann wieder gröfsere Partieen in kurze Sätze zusammengezogen hat. Die Möglichkeit einer solchen Unregelmälsigkeit der Kürzungen ist auch für unsere beiden Redaktionen zuzugeben, wie denn auch innerhalb der beiden kontrollierbaren Partieen die Veränderungen unregelmäfsig verteilt sind. In dem ersten Abschnitt (s. unten) sind die Veränderungen viel gewaltsamer und tiefgreifender als im zweiten. Im Übrigen liegt auf der Hand, wie verschieden in dem Grade der Veränderungen diese Redaktoren und jener Epitomator gearbeitet haben. Auch nach dem Funde des Codex Thebanus können 
wir doch daran festhalten, dafs uns im Florentinus in der Hauptsache das Werk des Chariton erhalten ist, während in den Ephesiaca ganze Partieen übersprungen sind; nur haben wir kein Zutrauen mehr, dafs wir überall den vollen Wortlaut besitzen, dafs alle Bilder und Schmuckstückchen des Originals uns wortgetreu wiedergegeben sind. Es ist eine notwendige und verlockende Aufgabe, nunmehr den ganzen Text des Florentinus daraufhin zu untersuchen, an welchen Stellen sich die Hand des oben nachgewiesenen Redaktors erkennen läfst. Der Nachweis wird nicht so sicher zu erbringen sein, wie ihn Bürger für die Epitomierung des Xenophon erbracht hat, da im Florentinus die Änderungen eben nicht so durchgreifende sind. Diese Arbeit mufs ich Berufeneren überlassen; fürchte ich doch schon mit dem hier Gebotenen mich von meinem Studiengebiet zu weit entfernt zu haben.

Erst nach Abschlufs der obigen Untersuchungen bin ich darauf aufmerksam geworden, dafs ich für Chariton zu einem Resultat gekommen bin, das mit dem jüngst von Elimar Klebs für die historia Apollonii Tyrii gewonnenen grofse Ähnlichkeit zeigt. Auf Grund seiner ebenso mühseligen wie scharfsinnigen Behandlung der unendlich verwickelten handschriftlichen Tradition der historia ist Klebs (Die Erzählung des Apollonius aus Tyrus, Berl. 1899) zu dem Ergebnis gekommen, dafs uns als älteste Überlieferung der historia zwei von einander unabhängige Redaktionen der verlorenen christlichen Überarbeitung des lateinischen Urtextes erhalten sind. Doch während wir hier wegen der willkürlichen Umarbeitungen und starken Interpolationen nicht einmal die christliche Bearbeitung wieder herstellen können, scheint mir für Chariton der Versuch nicht aussichtslos, durch Ineinanderarbeitung der beiden Redaktionen sogar den Charitonischen Urtext - oder doch wenigstens etwas diesem sehr nahe kommendes - wiederherzustellen. Denn einerseits liegen hier nur Kürzungen vor, und es fehlen die Interpolationen, andererseits sehe ich kein Indicium dafür, dafs etwa schon die beiden Redaktoren von Th und $\mathrm{F}$ nur eine Überarbeitung des Chariton vor sich gehabt hätten. Ich möchte daher einstweilen, bis dieser Nachweis erbracht wird, annehmen, dafs der Text da, wo Th und F übereinstimmen - abgesehen natürlich von den überall vorauszusetzenden Schreibfehlern etc. - Charitons Worte bietet. Wo Th und F von einander abweichen, war es oft schwer, eine evidente Entscheidung über das Ursprüngliche zu treffen. Hier konnte ich nur Vermutungen geben; gründlichere Kenner dieser Litteratur werden vielfach anders urteilen. - In dem folgenden Versuch, den Chariton herzustellen, sind alle Konjekturen in spitze Klammern geschlossen. Die Begründungen sind meist oben gegeben. 
I. Abschnitt. Vgl. Hercher II S. 148, 19 ff.

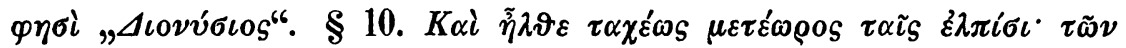

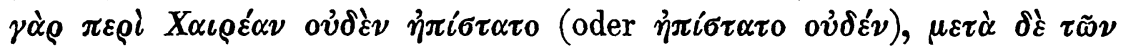

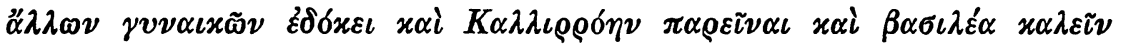

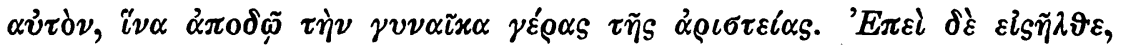

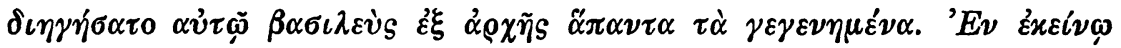

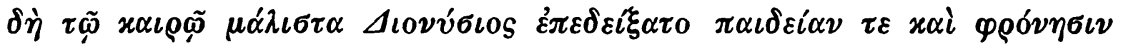

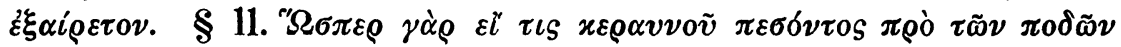

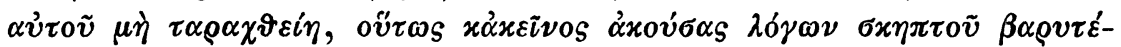

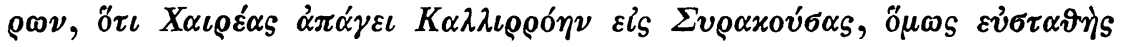

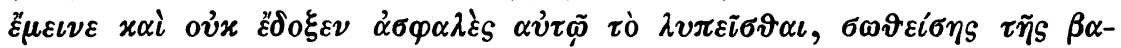

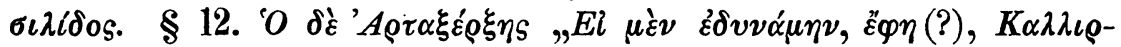

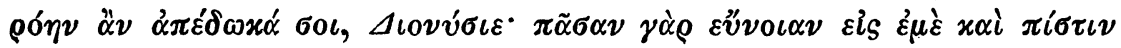

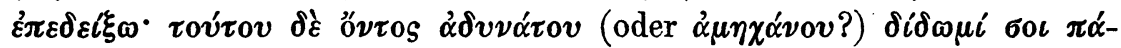

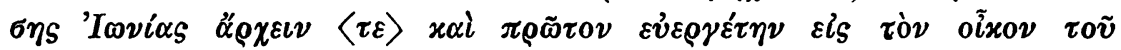

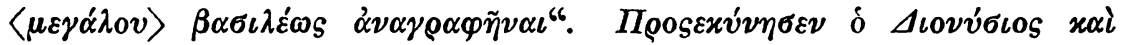

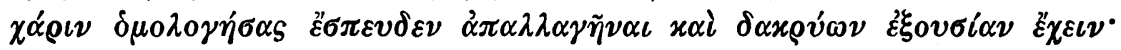

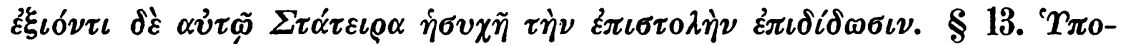

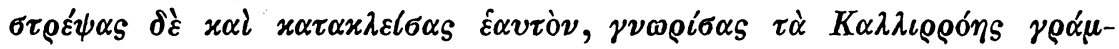

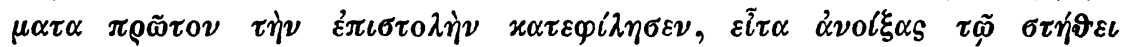

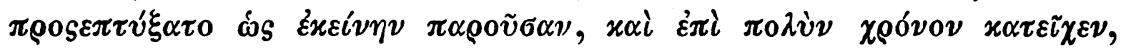

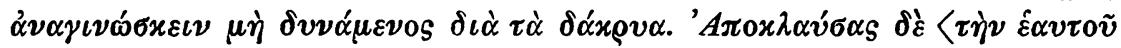

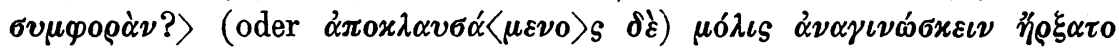

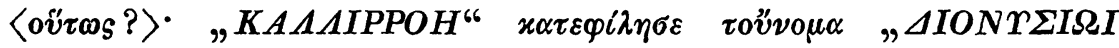

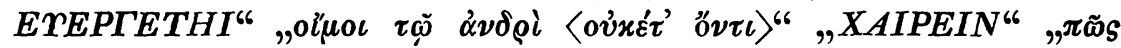

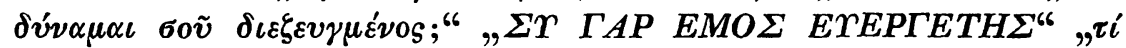

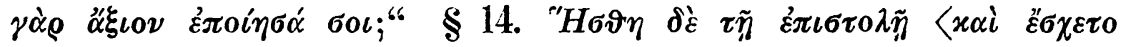

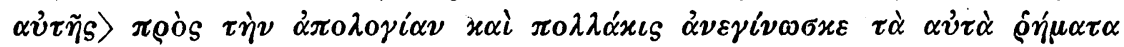

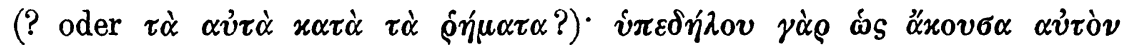

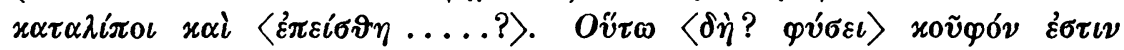

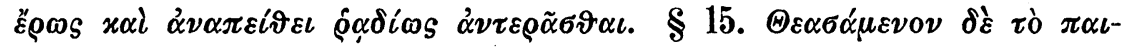

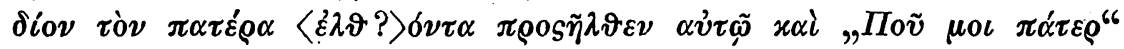

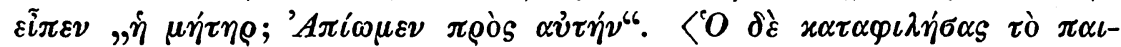

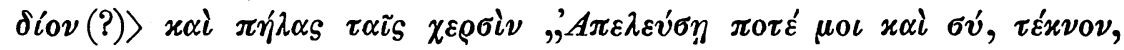

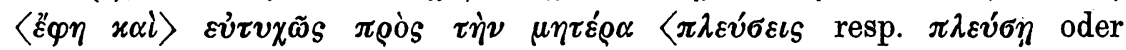

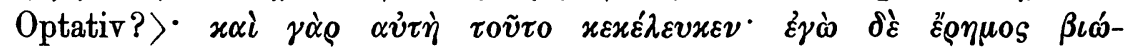

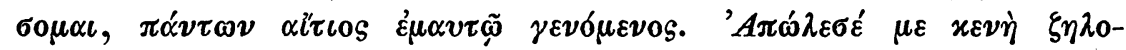

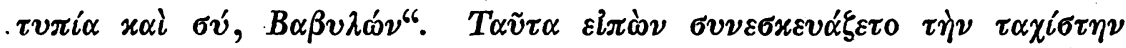

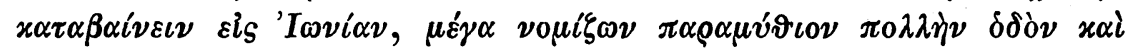

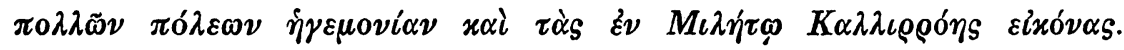




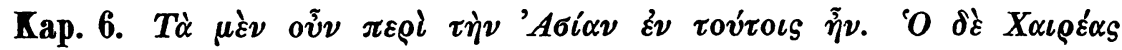

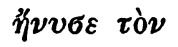

\section{Abschnitt. Vgl. Hercher S. 151, 13 ff.}

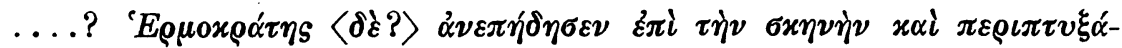

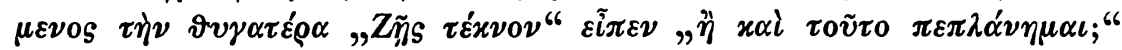

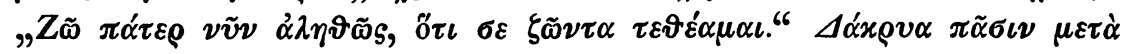

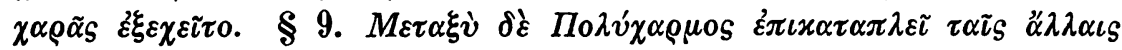

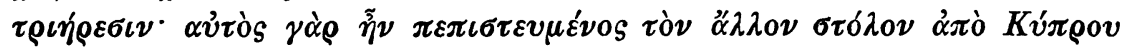

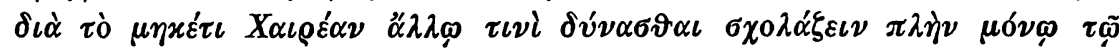

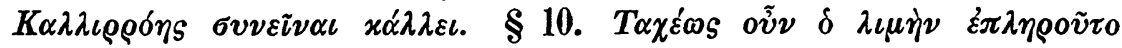

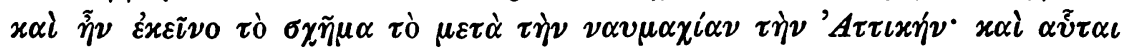

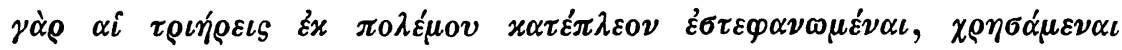

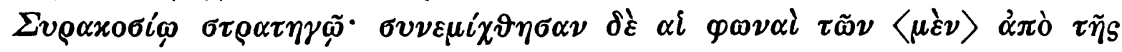

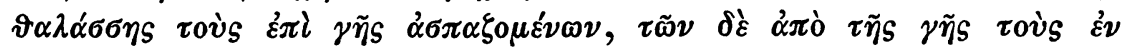

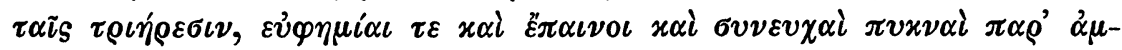

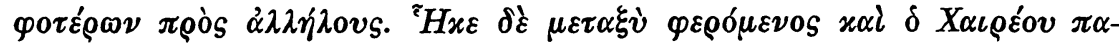

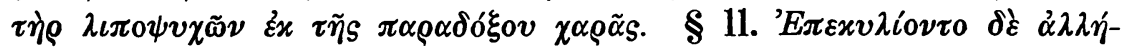

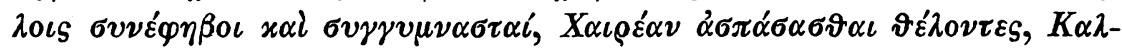
$\lambda \iota \varrho \varrho o ́ \eta \nu ~ \delta \grave{\varepsilon} \alpha i$

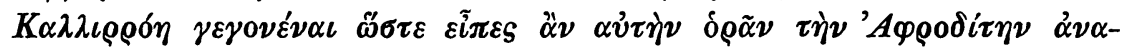

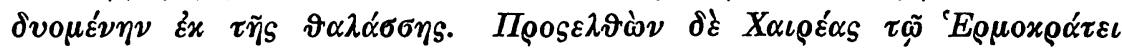

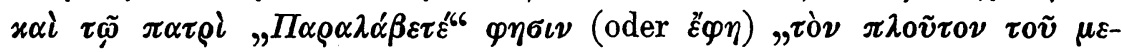

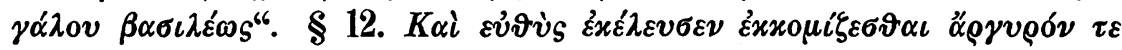

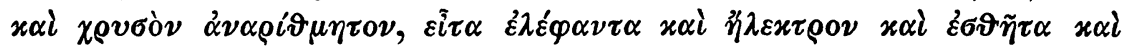

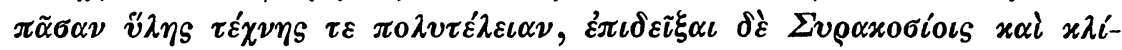

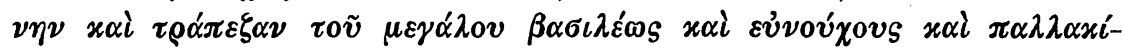

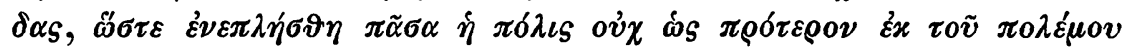

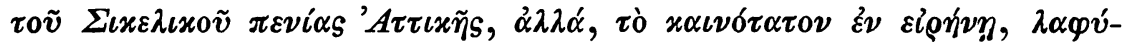

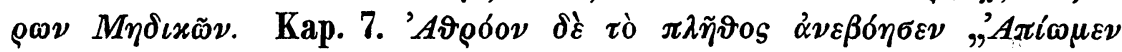

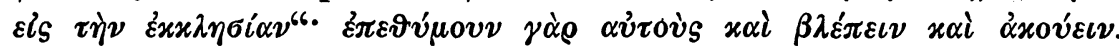

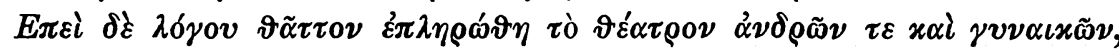

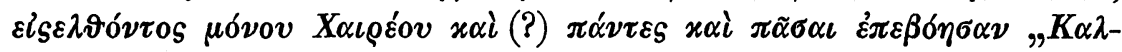

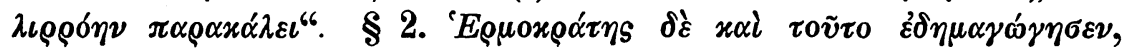

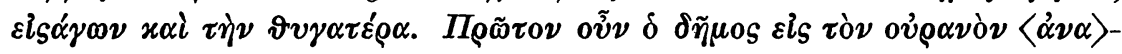

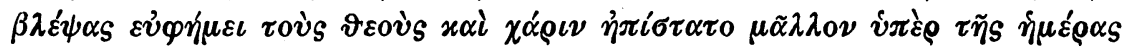

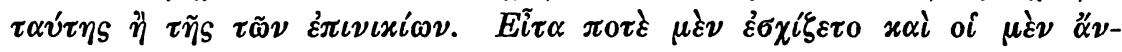

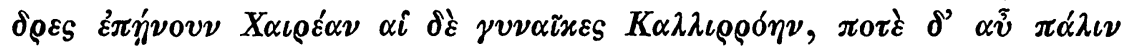

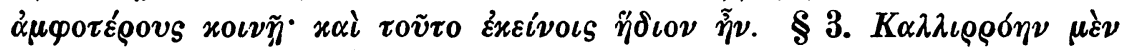
oี่ 


\section{Der Chione-Roman.}

Die Fragmente des neuen Romanes kann ich nicht mit derselben Sicherheit wie die des Chariton vorlegen. Abgesehen davon, dafs mir hier die Hilfe einer Edition fehlte, habe ich auch weniger Zeit auf diese beiden Blätter verwendet. Trotz der Unsicherheit mancher Lesungen will ich meine Kopie im Folgenden mitteilen, da es litterarhistorisch doch nicht uninteressant ist, einen neuen Roman, wenn auch nur in dürftigen Fragmenten, kennen zu lernen.

Ich bedauere, über die Anordnung der drei Fragmente mir nichts notiert zu haben. Zwei von ihnen müssen ja sicher neben einander auf demselben Blatt gestanden haben, da ich, wie oben bemerkt, nur die Fleischseiten berücksichtigt habe, also nur vier Kolumnen in Betracht kommen. Es ist lediglich ein nachträglicher Schlufs aus dem Inhalt, wenn ich annehme, dals Kol. I und II in dieser Reihenfolge neben einander auf dem ersten Blatt gestanden haben. Wohin das dritte Fragment gehört, darüber unten eine Vermutung.

\section{Fragment $I$.}

$[\ldots \ldots \ldots \ldots] \alpha_{s} \dot{\eta}$

$[\beta] \alpha \sigma \iota \lambda \varepsilon i \alpha$ cls $\tau \alpha \dot{v} \tau \eta \nu$

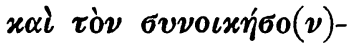
$\tau \alpha \alpha \dot{v} \tau \tilde{\eta} \mu \varepsilon \tau \dot{\varepsilon} \rho \chi \varepsilon-$

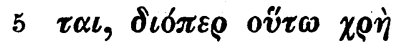
$\beta 0 v \lambda \varepsilon v \sigma \alpha \sigma \sigma \alpha \iota \nu \tilde{v} \nu$ $\hat{\eta} \mu \tilde{\alpha} S$ ஸ́s $\mu \grave{\eta} \mu \varepsilon \tau \alpha-$

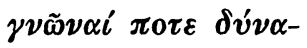

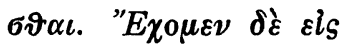

10

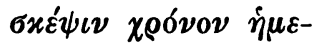

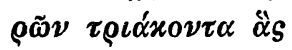

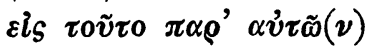

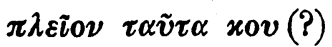
Darauf fehlen 15 Zeilen.

Fragment II.

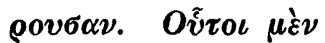

$\tilde{\eta} \sigma \alpha \nu \pi \rho \grave{\partial}\langle s\rangle \tau[\tilde{\omega}] \pi \varepsilon \rho[i \quad \alpha \dot{v}]-$

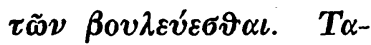
$\chi \varepsilon \dot{\varepsilon} \omega s \quad \delta \dot{\varepsilon} \delta\llcorner\varepsilon \varphi \circ i ́ \tau \eta \sigma \varepsilon$

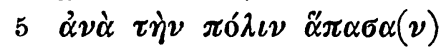

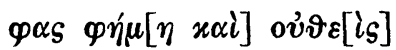

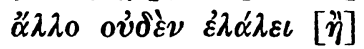

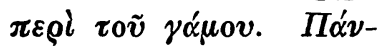

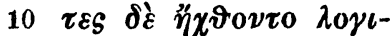

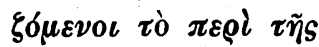

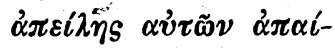

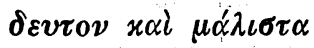

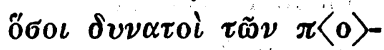

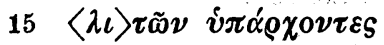

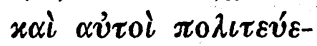

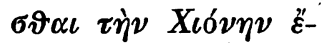
$\mu \varepsilon \lambda \lambda o v \mu \nu \eta \sigma \tau \varepsilon v^{\prime} \varepsilon-$

II $2 \pi \rho \circ \varepsilon \tau . \pi \varepsilon \rho$ Kopie. - II 14/5 $\pi \alpha \mid \nu \tau \omega \nu$ Kopie. Dies nicht nur sachlich, sondern auch paläographisch unwahrscheinlich, da $\pi \alpha v \mid \tau \omega \nu$ getrennt worden wäre. Die Verlesung von $N$ statt $A I$ liegt sehr nahe. 


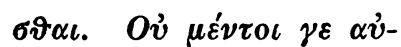

20

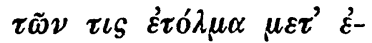

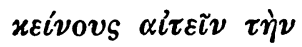

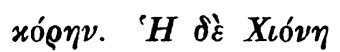

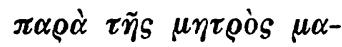

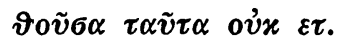

Darauf fehlen 4 Zeilen.

\section{Fragment III.}

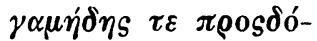

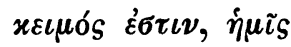

$\delta \dot{\varepsilon} \mu \varepsilon \dot{\varepsilon} \chi \varrho \iota \tilde{v} \nu \pi \alpha \dot{\alpha} \nu \tau \alpha$

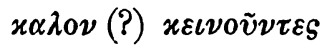

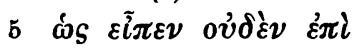

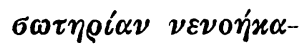

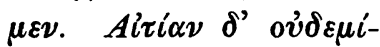

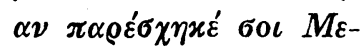

$\gamma \alpha \mu \eta \dot{\delta} \eta \bar{s}$ i" $\nu \alpha \dot{\alpha} \pi 0 \lambda i-$

$10 \pi \eta \varsigma \alpha \dot{v} \tau o ́ v$. "$\Omega \sigma \tau \varepsilon \delta\llcorner\alpha-$

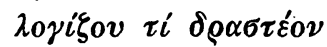

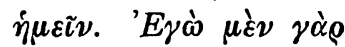

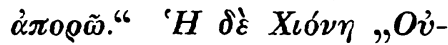

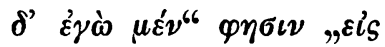

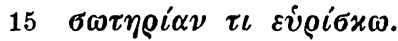

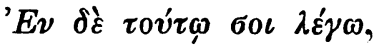
$\varepsilon i \mu \grave{\eta} \delta v \nu \alpha \dot{\mu} \varepsilon \varepsilon \vartheta \alpha \xi \tilde{\eta}(\nu)$ $\mu \varepsilon \tau ' \dot{\alpha} \lambda \lambda \dot{\eta} \lambda \omega \nu, \pi \rho \circ \sigma \tau \alpha^{\prime}-$ $\xi_{\alpha} \alpha \tau \varepsilon \varepsilon \varepsilon_{S} \tau o v \tau \omega \quad \varepsilon \pi$.

20 $\delta v \ldots \ldots \ldots \ldots \ldots$

.......... Tho jò

$\tau \varepsilon \lambda \varepsilon v \tau \alpha i \tilde{o} \nu \dot{\eta} \mu[\tilde{\imath} \nu]$

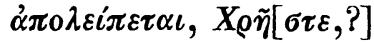

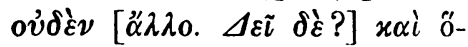

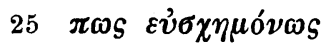


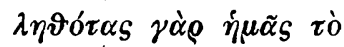

Versuchen wir, diese disiecta membra zu verstehen.

Fragment I führt uns in eine Versammlung von Männern, die miteinander beraten. Der Redner sagt: „die Königsherrschaft [soll] auf diese [übergehen (?)] und er beruft ihren künftigen Gemahl. Darum müssen wir jetzt derartige Beschlüsse fassen, dafs wir niemals Reue empfinden können. Wir haben zur Überlegung dreilsig Tage Zeit" das Weitere verstehe ich nicht.

Nach Frg. II ist es nicht zweifelhaft, dafs diese Königstochter,

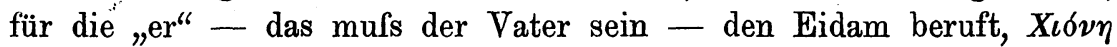
ist. Wir haben also einen Roman vor uns, der die Schicksale einer schönen Prinzessin behandelt. Die meisten der uns erhaltenen griechischen Romane lassen ihre Liebespaare in vornehmen, aber bürgerlichen Kreisen aufwachsen. Aufser dem Ninosroman bietet noch die historia Apollonii Tyrii Parallelen zu dem unsrigen: dieser handelt im Anfang von der Tochter des Königs von Tyros, an deren Stelle dann bald die Königstochter von Kyrene in den Mittelpunkt tritt.

$X \iota \delta$ v heifst die Prinzessin. Gewifs wird der Dichter die Spielerei mit dem Namen sich nicht haben entgehen lassen, sondern wird den

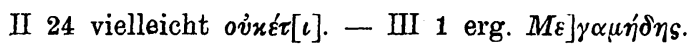


weifsen Teint dieses "Schneewittchen" gebührend hervorgehoben haben. Vgl. ähnliches bei Rohde, Gr. Rom. S. 153. Der Name Xıóv $\eta$ eignet, abgesehen davon, dafs er auch als Frauenname vorkommt, mehreren Gestalten der griechischen Sage, einer Nymphe, einer Tochter des Boreas u. s. w., auch jener Tochter des Daidalion, deren Liebesgeschichte Ovid (Metamorph. XI $301 \mathrm{f}$.) mit den Worten beginnt: Nata erat huic Chione, quae dotatissima forma mille procos habuit, bis septem nubilis annis. Mir ist aufgefallen, dafs auch sonst Namen von Heldinnen griechischer Romane in den Sagen als Namen von Nymphen und ähn-

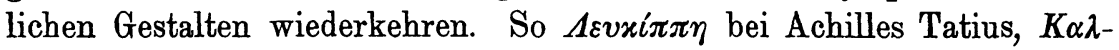
$\lambda \iota \varrho \varrho o ́ \eta ~ b e i ~ C h a r i t o n, ~ \Pi \alpha \varrho \vartheta \varepsilon v \delta \pi \eta$ in dem Berliner Fragment (s. unten S. 264 ff.). Sollten nicht trotz des rein menschlichen Charakters dieser Romane die betreffenden Sagengestalten den Romanschriftstellern vorgeschwebt haben? Freilich würde schliefslich nicht viel mehr als der Name übrig geblieben sein, denn dem Romanschema zu Liebe mülsten alle ursprünglichen sagenhaften Züge von jenen Gestalten genommen worden sein. Vielleicht ist es für die Entwickelungsgeschichte der Romane nicht ohne Bedeutung, dafs der älteste uns bekannte, der Ninosroman, trotz starker Änderungen, die dem Romanschema zu Liebe auch schon bei ihm vorgenommen sind (vgl. Hermes 28), doch noch einige Züge der zugrunde liegenden Sage, wie z. B. Ninos als Welteroberer, deutlich bewahrt hat. Da dieser Ninosroman aber sicher nicht das erste Beispiel der neuen Gattung gewesen ist, sondern schon seine Vorläufer gehabt haben mufs, so liegt der Gedanke nahe, dals vielleicht die ältesten selbständigen erotischen Erzählungen in Prosa, deren Kunde uns verloren ist, sich inhaltlich noch enger an die Sagen, wie sie in der hellenistischen Dichtung und andererseits gelegentlich von den Historikern ${ }^{1}$ ) verarbeitet waren, angeschlossen haben, dal's bei den Späteren dann das Sagenhafte immer mehr geschwunden ist, bis zuletzt nur noch die Namen an den ursprünglichen Ausgangspunkt erinnerten.

Doch kehren wir nach dieser Hypothese wieder zur Chione zurück. Aus den ersten Worten des Fragmentes ergab sich, dafs ihr Vater für

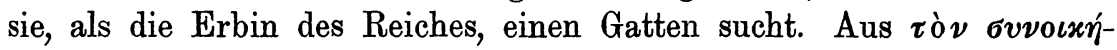
бov $\tau \alpha$ möchte ich nicht folgern, dafs er eine bestimmte Persönlichkeit

1) Vgl. die sehr anregenden Ausführungen von Ed. Schwartz, Fünf Vorträge über den griechischen Roman. Berlin 1896. - Nach Sehwartz ist es für die erotischen Erzählungen gerade charakteristisch, dals ihre Personen nicht aus Sage oder Geschichte genommen, sondern frei erfunden sind. Aber wo bleibt da Metiochos und Parthenope? oder Ninos und Semiramis? 
herbeiruft; vielmehr scheint er verkündet zu haben, dafs er unter den und den Bedingungen nach Ablauf von dreifsig Tagen seine Tochter vermählen wolle. Daraufhin haben sich die Männer, in deren Beratung wir in Frg. I eingeführt werden - vielleicht der höchste Adel -, versammelt. Nach Frg. II haben sie selber Freiergelüste und vielleicht haben sie sich verbündet, um durchzusetzen, dafs einer von ihnen die Braut heimführe und das Reich gewinne (vgl. II $19 \mathrm{ff}$.). Ist diese Auffassung zutreffend, so würden wir dadurch an den Anfang des Charitonromanes erinnert werden, wo die abgewiesenen Freier - allerdings nach der Hochzeit - sich zu gemeinsamen Beratungen zusammenfinden (I 2,1):

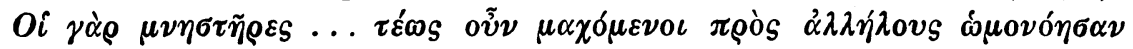

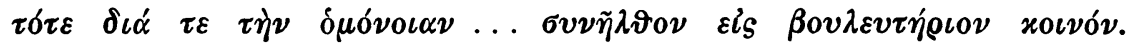
Darauf folgen wie hier ausführliche Reden der Beratenden. Für die oben angenommene Situation hebe ich folgende Worte daraus hervor:

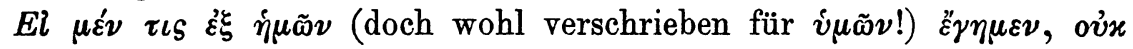

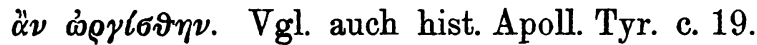

Die dreifsigtägige Frist ist nicht ohne Interesse, denn sie gehört zu den üblichen Requisiten der griechischen Romane. Man vergleiche

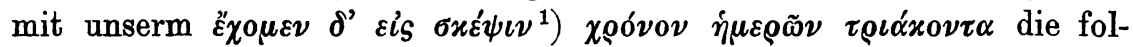
genden Stellen: Xenoph. II 13, 8: der Irenarch Perilaos drängt die

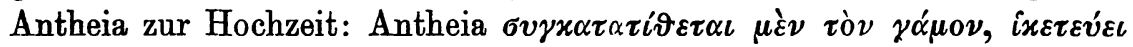

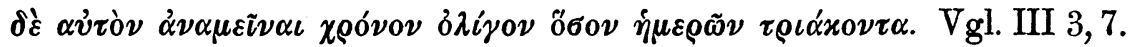

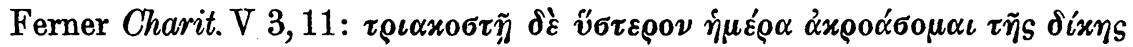

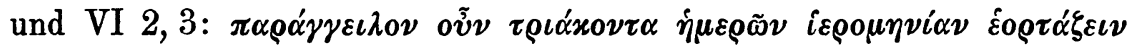

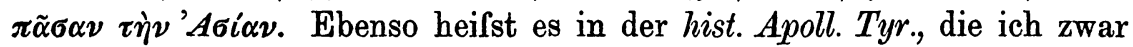
nicht für eine Übersetzung, wohl aber für eine freie Bearbeitung eines ursprünglich wohl viel ausführlicheren griechischen Originalromanes halte ${ }^{2}$ ), in c. 5 folgendermalsen: sed habebis triginta dierum spatium: recogita tecum.

1) Vgl. Charit. II 10, 7; VI 7, 13.

2) So ungern ich nach nur vorübergehender Beschäftigung mit dieser Frage einem Forscher widerspreche, der mehrere Jahre seines Lebens diesen Problemen gewidmet hat, möchte ich es hier doch aussprechen, dals die Beweisführung von Elimar Klebs (vgl. oben S. 252) mich nicht davon überzeugt hat, dafs wir in der hist. Apoll. ein lateinisches Originalwerk vor uns haben. Er giebt zwar die Möglichkeit zu, dafs der Verfasser sich in Einzelheiten an griechische Vorbilder angelehnt habe (S. 298. 306), hält das Ganze aber doch, da hier und da die Benutzung von lateinischen Vorbildern wie Vergil und Ovid nachgewiesen ist, für „ein lateinisches Originalwerk in dem Sinne, in welchem diese Bezeichnung den Werken der römischen Dichtung überhaupt zukommt" (S. 307). Wiewohl die wirklichen oder angeblichen "römischen" Elemente in der hist. Apoll. für die Hauptfrage m. E. gar nicht entscheidend sind, sei doch darauf hingewiesen, dals 
Der Bericht über die Versammlung jener Männer, der in II 1

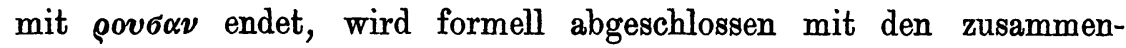

nicht alle seine Nachweisungen römischer Elemente überzeugend sind. So geht K. entschieden zu weit, wenn er S. 211 in den Worten (c. 7) alia vero die in civitate sua quaeritur a civibus suis ad salutandum eine Anspielung auf den Morgenbesuch beim Kaiser und den Vornehmen sehen will. Denn was ist natürlicher, als dafs die Bürger ihren princeps nach seiner Heimkehr begrülsen wollen? Vgl. übrigens zu jener römischen Sitte meine Bemerkungen über den $\alpha \sigma \pi \alpha \sigma \mu o ́ s$ am Ptolemäerhofe in Berl. Phil. Woch. $1896 \mathrm{Sp}$. 1462. Andrerseits scheinen mir die engen Beziehungen zu den griechischen Romanen nicht scharf genug herausgehoben zu sein. Wenn z. B. Apollonius zum Schlufs sein Leben aufzeichnet, ein Exemplar für sich behält, ein anderes im Tempel von Ephesos aufbewahrt, so trifft der Hinweis auf den M. Antonius, der sein Testament bei den Vestalinnen deponiert habe sowie auf Heraklit (S. 211), eine Nebensache. Viel wichtiger ist an dieser Geschichte, dafs derselbe Grundgedanke, dals die Erzählung auf urkundliches autobiographisches Material zurückgehe, fast mit denselben Details sich am Schlufs des griechischen Romanes des Antonius Diogenes wiederfindet: auch dort schreibt der Held (durch einen Redekünstler) schliefslich sein Leben auf zwei Tafeln nieder, von denen eine $K v ́ \mu \beta \alpha$ f für sich behalten soll, während die andere später in seinem Grabe niedergelegt werden soll. Dagegen erweckt der Hinweis auf die römischen Grofsen und Fürsten, die seit Sulla ihre Autobiographien geschrieben hätten (S. 208), die irrige Vorstellung, als wenn das etwas speziell Römisches sei. Ein ander Mal bemerkt K. (S. 289) zu der Erzählung, dals Apollonius in Verzweiflung über den Tod seiner Gattin klagend im untersten Sehiffsraume gelegen habe: „Nirgends begegnet das Gleiche bei den griechischen Romanschreibern" - was würde das übrigens beweisen, wo jetzt die Funde der letzten Jahre uns vor Augen geführt haben, wie gering unsere Kenntnis dieser Litteratur ist? - und verweist auf zwei Stellen bei Apuleius, die doch nur wieder in Nebensächlichem mit der hist. übereinstimmen. Da hat er übersehen, dafs eine viel bessere Parallele sich in dem

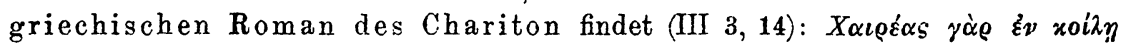

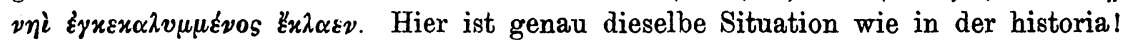

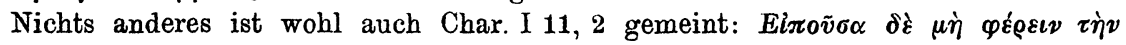

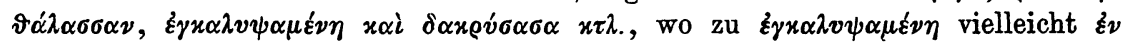
xoíly $v \eta$ hinzuzudenken ist. Die Hinweisungen auf Apuleius hier und an anderen Stellen verlieren übrigens vielfach an ihrer Beweiskraft für den römischen Ursprung der historia, nachdem soeben von Dietze (Philol. N. F. XIII S. $136 \mathrm{ff}$.) erwiesen ist, dafs auch der apulejischen Erzählung von Amor und Psyche ein griechisches Original zu Grunde liegt. - Doch zugegeben, dals mehrere Züge in der historia speziell auf römische Verhältnisse hinweisen, so ist das ebenso wie die Benutzung von Vergil und Ovid durchaus mit der Annahme vereinbar, dafs wir eine lateinische Überarbeitung eines griechischen Originals vor uns haben: ebenso wie der Bearbeiter für sein lateinisches Publikum statt der griechischen die römischen Münzen eingeführt hat, so hat er auch statt der griechischen Dichtercitate, mit denen - meist aus Homer - die griechischen Romane durchsetzt sind, seine römischen Dichter hineingearbeitet, und ebenso gut kann er auch hier und da einen römischen Zug hineingebracht haben. - Ebenso wenig wie der obigen 


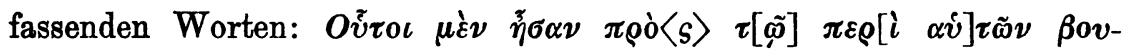

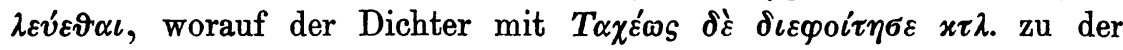
Schilderung des Eindrucks übergeht, den die Nachricht von der bevorstehenden Hochzeit der Königstochter in der Stadt hervorruft. Das ist die auch in den anderen Romanen übliche Art der transitio. Bürger hat kürzlich diese Form des Übergangs im besonderen für die Ephesiaca des Xenophon ausführlich nachgewiesen (Hermes 27, $51 \mathrm{ff}$.). Aber auch in den anderen Romanen finde ich beim Durchblättern überall Beispiele. Vgl. Charit. I 11, 4; VII 6,1; VIII 6, 1 .

Auch die Art, wie die schnelle Ausbreitung des Gerüchtes in der Stadt geschildert wird, findet in den anderen Romanen ihre Parallelen. Vgl. Achill. Tat. VI 10, 3: 'H $\delta \dot{\varepsilon} \varphi \eta^{\prime} \mu \eta \delta\llcorner\alpha \pi \varepsilon \varphi o i \tau \eta x \varepsilon \nu ~ \varkappa \tau \lambda$. Vgl. ferner

Schlufsfolgerung vermag ich mich der anderen Ansicht von $K$. anzuschliefsen, dafs die historia weder von Abenteurerfahrten noch von Liebesgeschichten genug bringe, um sie nach der Rohde'schen Definition inhaltlich überhaupt den griechischen Romanen gleichzustellen (S. 295-298). Mir scheint nach beiden Seiten hin, selbst in der dürftigen Form, in der die Geschichte uns jetzt vorliegt, vollauf genug vorhanden zu sein. Wenn Apollonius von Tyros nach Tarsos, von dort nach Kyrene, dann wieder nach Tarsos geht, darauf nach 14jährigem Umherstreifen in Ägypten (!) nach Tarsos zurückkehrt, dann durch einen Seesturm nach Mytilene verschlagen wird, um endlich über Ephesos, Antiochien, Tyrus, Tarsos wieder nach Kyrene zu kommen, so ist das Moment des abenteuerlichen Umherziehens, wenn auch nicht so ausgebildet wie bei Xenophon oder Heliodor, so doch - von Longus ganz abgesehen - noch stärker als z. B. in den Babyloniaca des Jamblichos, wo das Liebespaar aus der Euphrat-Tigris-Ebene kaum herauskommt. Und was die Erotik betrifft, so ist sie darum nicht schwächer, weil - von der Antiochus-Episode ganz abgesehen - die üblichen Liebesbedrängnisse hier auf Mutter und Tochter verteilt sind. Wenn K. z. B. die Gefährdung der Keuschheit bei der Mutter vermifst, so darf doch nicht übersehen werden, dafs dieser beliebte Romanzug in der Geschichte der Tochter, die selbst im Bordell sich rein erhält, mit kaum zu überbietender Klarbeit zum Ausdruck kommt. So möchte ich trotz K.s Forschungen an der herrschenden Meinung, dals der historia ein griechischer Roman zu Grunde liege, festhalten. Das griechische Originalwerk mag ursprünglich einen Umfang wie der Durchschnitt der uns erhaltenen sophistischen Liebesromane gehabt haben, liegt also, wie mir scheint, in starker Epitomierung vor. Dals z. B. im griechischen Original der 14jährige Aufenthalt in Ägypten, der so schöne Veranlassung zu den buntesten Abenteuern gab, nicht ausführlicher dargestellt sein sollte, ist mir im höchsten Grade unwahrscheinlich. Auch viele Unklarheiten der historia würden durch diese Annahme ihre beste Erklärung finden. $\mathrm{Ob}$ erst der lateinische Bearbeiter diese Epitomierung vorgenommen, oder ob er bereits eine griechische Epitome überarbeitet hat, mögen andere entscheiden. Der schlichte, volkstümliche Ton, der die Dichtung zu einem der beliebtesten Volksbücher im Abendlande gemacht hat, ist gewils erst durch die Epitomierung - vielleicht ungewollt - hineingekommen. 
Chariton I 1, 2; I 5, 1; Xenoph. I 12,1. Ähnlich mag es auch in dem griechischen Original zur hist. Apoll. c. 49 geheifsen haben, wo wir jetzt lesen: sonat in tota Epheso, Tyrium Apollonium recognovisse suam coniugem etc., womit man noch Charit. VIII 1, 11 vergleichen möge:

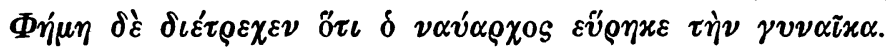

Aus den Reflexionen der städtischen Berölkerung, die uns im Folgenden mitgeteilt werden, erfahren wir noch Genaueres über das Vorgehen jener beratenden Männer. Denn auf sie beziehen sich doch

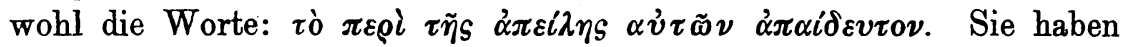
also in roher Weise Drohungen ansgestofsen - wohl schon vor jener (geheimen?) Beratung. Diese Drohungen beziehen sich auf die bevorstehende Werbung um Chione, und wenn es Z. $19 \mathrm{ff}$. heifst, dafs keiner

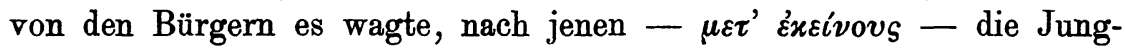
frau für sich zu fordern, so war mir das ein Grund für die oben ausgesprochene Vermutung, dafs jene Beratung eben den Zweck hatte, einem von ihnen die Königstochter zu verschaffen.

Jene Drohungen, die offenbar gegen etwaige Konkurrenten ausgestofsen worden sind, werden von der Bevölkerung mit Entrüstung

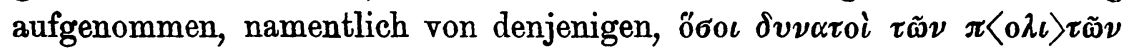

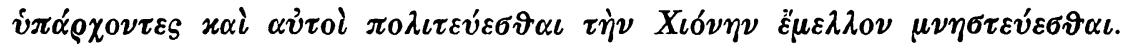
Diese nicht leicht verständlichen Worte werfen ein wenn auch schwaches Licht auf die Verkündigung des Königs betreffs der Bewerbung um

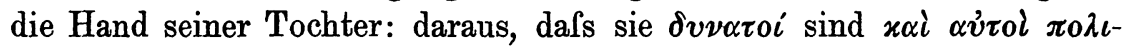
$\tau \varepsilon v_{\varepsilon} \sigma \vartheta \alpha \iota$ wird die Erwartung abgeleitet, dals sie sich um Chiones Hand bewerben würden ( $\left.\varepsilon_{\mu} \mu \varepsilon \lambda \lambda o \nu !\right)$. Also war das wohl eine condicio sine qua non für die Bewerbung, dals man Bürger war und fähig war, auch selbst sein Bürgerrecht auszuüben, oder seinen Bürgerpflichten obzuliegen, oder wie man das vieldeutige $\pi 0 \lambda \iota \tau \varepsilon v \varepsilon \sigma \vartheta \alpha \iota$ fassen will. In dieser Forderung des Bürgerrechts erkennt man den griechischen Autor! Wir erfahren leider nicht, wie aus dieser grofsen Zahl von Bewerbern der eine Glückliche ausgewählt werden sollte, ob etwa Chione - wie Odatis in dem schönen Märchen vom Zariadres (Chares fr. 37 Müller) selbst einen aussuchen, oder ob der Freier sich etwa durch eine besondere Leistung - wie die Rätsellösung in der historia Apollonii als der Rechte erweisen sollte.

Zum Schlufs von Frg. II erfahren wir dann, dafs Chione über diese aufregenden Vorgänge durch ihre Mutter benachrichtigt wird. Wahrscheinlich wird eine sehr thränenreiche Unterhaltung zwischen Mutter und Tochter gefolgt sein, ähnlich der zwischen der Geliebten des Ninos und ihrer Tante Thambe (Hermes 28 S. 172). Denn dafs das Herz der Jungfrau schon gewählt hatte, und zwar den Wünschen 
des Vaters entgegen, dürfen wir aus dem III. Frg. schliefsen, und wird auch von dem üblichen Romanschema gefordert.

Das III. Frg. ist am schwersten zu verstehen. Ich habe es zunächst unter der Voraussetzung zu deuten gesucht, dafs das zweite Blatt im Codex unmittelbar auf das erste gefolgt sei, so wie im Charitonroman die Blätter auf einander folgten. Dies ergab mir aber keinen befriedigenden Sinn. Namentlich bleibt mir dann unverständ-

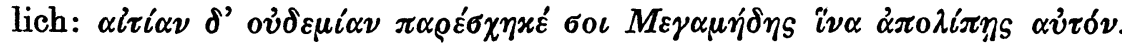
In welchem Verhältnis sollte in dem bisher ins Auge gefalsten Zeitpunkt Chione zu diesem Megamedes gestanden haben? Von ihrem Vater würde kaum so gesprochen werden, sondern es würde wohl $\delta$ $\pi \alpha \tau \eta \dot{~ \sigma o v ~ g e s a g t ~ s e i n . ~}$

Es zwingt aber nichts zu der Annahme, dafs die Blätter unmittelbar auf einander gefolgt seien; wir können ebenso gut annehmen, dafs das zweite Blatt einem späteren Teil des Codex angehört hat. Unter dieser Voraussetzung schlage ich folgende Erklärung vor.

Frg. III enthält ein Zwiegespräch zwischen Chione und einem

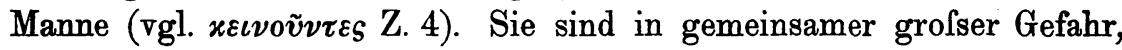
suchen nach Rettung, und Chione erklärt ihm, nicht ohne ihn leben zu können. Kurz, der Mann, dessen Rede Z. 1-13 angehören, ist niemand anders als der Geliebte der Chione, der Held des Romanes.

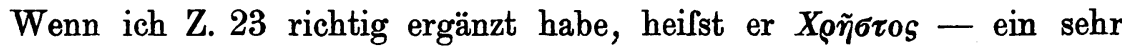
passender $\mathrm{Name}^{1}$ ) für den Liebhaber, der ja in diesen Romanen ein Tugendbold zu sein pflegt. Vielleicht gehörte er nicht zu jenen $\pi 0^{-}$ $\lambda i \tau \alpha \iota$, die zu der Werbung berechtigt waren. Wer ist dann $\boldsymbol{M} \varepsilon \gamma \alpha-$

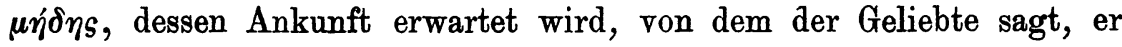
habe der Chione keine Veranlassung gegeben, dals sie ihn verlasse? Ich denke, das ist der, der als Sieger aus dem Wettbewerb um Chiones Hand hervorgegangen ist, ihr Gemahl. Oder ist er noch nicht ihr

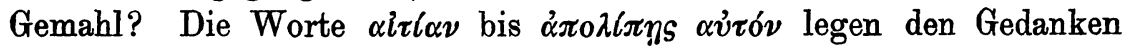
nahe, dafs sie schon bei ihm war, ihm gehörte. Andrerseits würde die Rede der Chione wie überhaupt die ganze Situation klarer, wenn man annehmen dürfte, dafs dies Gespräch etwa unmittelbar vor der Hochzeit gehalten wäre. Ich lasse die Frage unentschieden.

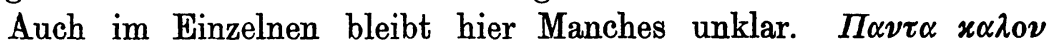

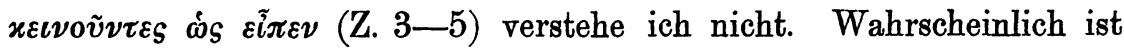

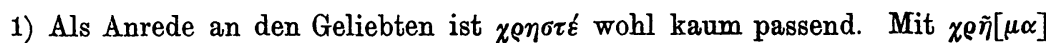
wülste ich hier nichts anzufangen. - Dafs die Namen gern eine Anspielung auf den Charakter enthalten, hat schon Rohde hervorgehoben. 


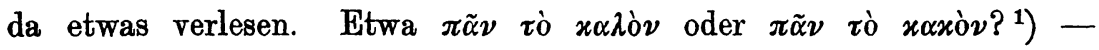
In der Lücke Z. 20/1 wird kaum etwas anderes gestanden haben, als die ultima ratio: "so wollen wir sterben mit einander" 2). - Die Ergänzungen von Z. $21 \mathrm{ff}$. gebe ich mit "um so gröfserem Vorbehalt, als ich sie nicht angesichts des Originals, sondern erst jetzt am Schreibtisch gemacht habe. Ich kann daher nicht aussagen, ob die vorgeschlagenen Ergänzungen zu den Schriftspuren passen.

Die Richtigkeit der Ergänzungen vorausgesetzt würde der Gedanken-

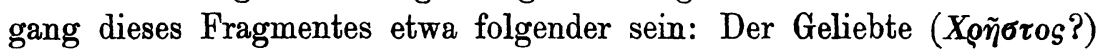
spricht: „die Ankunft des Megamedes ist zu erwarten, und wir .... haben noch nicht an unsere Rettung gedacht. Megamedes hat dir keine Veranlassung gegeben, dals du ihn verlassest. Drum überlege, was wir thun sollen, denn ich bin ratlos". Nachdem er so aus Edelmut gegen den edeln Megamedes, der ebenso wie Chrestos seinem Namen Ehre macht, den Gedanken an gemeinsame Flucht abgelehnt hat, zieht Chione die Konsequenz, indem sie sagt: „Da wir nicht mit einander leben können ... . [wollen wir sterben]. Denn schliefslich bleibt uns nichts anderes übrig. Wir müssen nur sehen, dafs wir auch auf vornehme Weise aus dem Leben gehen ...".

Der letzte Gedanke begegnet ähnlich bei Charit. VII 1, 7, wo

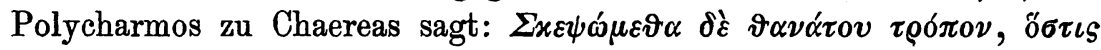

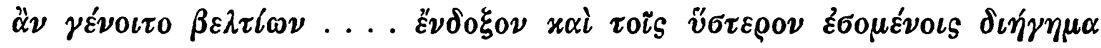
$x \alpha \tau \alpha \lambda \varepsilon i \pi 0 \nu \tau \alpha s \quad x \tau \lambda$.

Es ist nicht viel, was uns diese dürftigen Überreste, die offenbar dem Anfang des Romanes angehören, über seine Anlage lehren. Doch sie genügen, uns zu zeigen, dafs auch dieser Roman in der Charakteristik der Personen, der Erfindung der Situationen und dem Stil der Darstellung, wenn er natürlich auch wie jeder Roman seine eigentümlichen Besonderheiten hat, doch im wesentlichen in denselben Geleisen fährt wie die uns schon bekannten Romane. Bezeichnend ist, dafs von den drei erhaltenen Kolumnen nicht weniger als zwei vollständig mit Reden ausgefüllt sind! Sicherlich haben wir nicht eine Epitome, sondern den ursprünglichen Text vor uns; höchstens Kürzungen im Stil der beiden Chariton-Redaktionen könnten als möglich zugegeben werden. Die zahlreichen oben hervorgehobenen Parallelen, durch die der neue Roman mit den alten verknüpft wird, ermöglichen nicht die Entstehung des Romanes im Vergleich zu den anderen zeitlich zu fixieren, was kein Wunder ist, wenn man bedenkt, dals sogar die

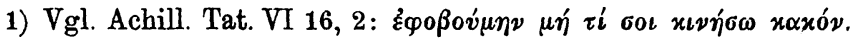

2) Vgl. das ähnliche Gespräch bei Xenoph. II 1. 
Zeitbestimmung der vollständig erhaltenen Romane vielfach auf sehr schwachen Füfsen steht. $\mathrm{Ob}$ der Chione-Roman zu den älteren oder den jüngeren gehört, lasse ich dahingestellt.

\section{Die ältesten Romane.}

Von dem Chione-Roman abgesehen sind uns in den letzten sieben Jahren durch ägyptische Papyrusfunde nicht weniger als drei neue griechische Romane bekannt geworden. So dürftig auch die Fragmente sind, haben sie doch durch ihr relativ hohes Alter - die Handschriften gehören dem I. resp. II. Jahrh. n. Chr. an! - ihre grofse Bedeutung für die Entwickelungsgeschichte des griechischen Romanes. Die weiten Zeiträume, durch die früher die hellenistische Erotik und die sophistischen Liebesromane getrennt waren (Rohde S. 165), werden nun durch diese neuen Funde, durch die wir Vorläufer der sophistischen Romane kennen lernen, überbrückt. $D a$ ich durch den thebanischen Palimpsest veranlafst wurde, auch diese Stücke von neuem zu prüfen, sei es mir gestattet, einige Beobachtungen, die sich mir dabei ergeben haben, kurz hier mitzuteilen. Nur den Ninosroman übergehe ich, da ich seit meiner Edition im Hermes 28 (1893) nicht wieder Gelegenheit genommen habe, das Original zu prüfen. So verweise ich nur auf die vielfach vortrefflichen Ergänzungen und Erläuterungen, die inzwischen von Ennea Piccolomini ${ }^{1}$ ) und Lionello Levi ${ }^{2}$ ) beigebracht worden sind.

Im Jahre 1895 edierte Krebs, gleichfalls aus den Schätzen des Berliner Museums, das Fragment eines Romanes, der die Liebesgeschichte von Metiochos und Parthenope erzählt (Hermes 30 S. $144 \mathrm{ff}$.). Im Anschlufs an diese Arbeit, die sich im wesentlichen auf die Textedition beschränkte, haben Kaibel und Robert ebendort das Fragment - es ist nur eine Kolumne - ergänzt und erklärt. Die Handschrift stammt, wie Krebs richtig geurteilt hat, aus dem II. Jahrh. n. Chr. Meine um Ostern an Krebs gerichtete Bitte, seine Edition nochmals am Original zu revidieren, sollte keine Erledigung mehr finden: der Tod hat ihn plötzlich von seinem Leiden erlöst. So habe ich selbst nachher das Original verglichen, und das Wenige, was sich für den Text ergeben hat, sei kurz hier mitgeteilt.

1) Rendiconti della R. Accademia dei Lincei 21. Mai 1893.

2) Rivista di Filologia e d' Istruzione Classica XXIII N. S. I 1894. 
Z. 1 l. $\tau] \dot{\eta} \underline{\underline{\nu}} \varphi[\iota \lambda] \underline{0 \sigma} \sigma \dot{\varphi} \sigma v \quad \xi \dot{\eta} \tau \eta \sigma \iota v$ :

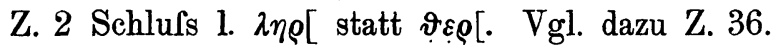

Z. 3 Schlufs sehe ich $\varepsilon \varphi o$, wodurch die Ergänzung von K-R '́ø' oís gestützt wird.

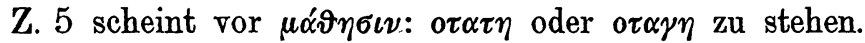

Z. 7 l. $[\tau \varepsilon] s$ o[i] $\tau \tilde{\eta}[s \quad \dot{\alpha} \lambda] \eta \vartheta \vartheta_{0} \tilde{v}_{s}$, wie K-R vermutet haben.

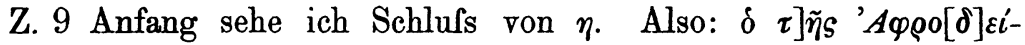

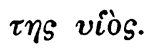

Z. 14 sehe ich $\sigma v \nu \underline{\varepsilon} \underline{\sigma} \underline{\tau} \eta \underline{x}[\varepsilon v$, wie K-R vermutet haben.

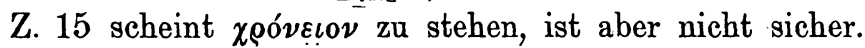

Z. 20 l. $\dot{\alpha} \pi \varepsilon \imath \vartheta \alpha v[o v$, wie K-R vermutet haben.

Z. 21 für die Ergänzung von $\mu o ́ v o] v$ scheint kein Platz zu sein.

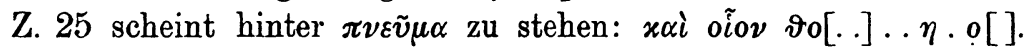
Bleibt mir unverständlich. Dagegen glaube ich das Folgende sicher erkannt zu haben:

Z. 25

$\ddot{i} \sigma \vartheta[\omega]-$

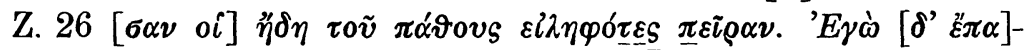

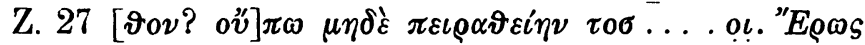

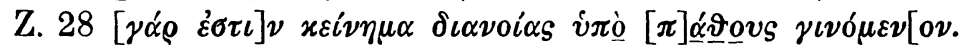
Hinter $\tilde{\varepsilon} \rho \omega s$ in Z. 27 braucht nichts zu fehlen.

Der lange Strich am Schlufs zwischen Z. 31/2, den R-K für die Paragraphos halten, gehört zu Z. 32: er tilgt die am Ende von Z. 32 geschriebenen Worte. Die Paragraphos mürste links, am Anfang stehen. Da der Papyrus sonst die Worte trennt, in Z. 32 aber $\varepsilon \omega S$ mit $\mu \varepsilon \lambda \varepsilon \tau \eta S$ eng verknüpft ist, dürfte $\mu \varepsilon] \lambda \varepsilon \tau \dot{\eta} \sigma \varepsilon \omega s$ zu verbinden

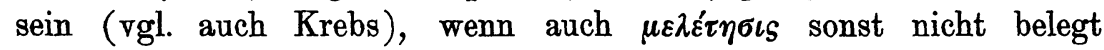
sein sollte.

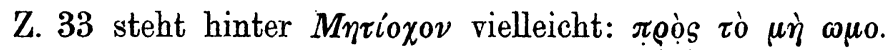

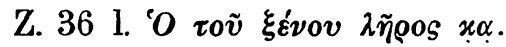

Wie schon Krebs S. 144 hervorgehoben hat, sind die beiden Romanhelden Metiochos und Parthenope offenbar dieselben, von deren Liebesgeschichte Eustathios zu Dionys. Perieg. 358/9 erzählt. Freilich ist a priori anzunehmen, dafs im Roman die Sage sehr stark verändert worden ist. Denn der Abschlufs, den Eustathios mitteilt (Parthenope entstellt sich und entsagt dem Geliebten), palst nicht zum griechischen Roman, der kein anderes Ende als die glückliche Vereinigung der Liebenden kennt. Auch die andere Sage, die Lucian de saltat. 2 kennt, wonach Parthenope zu den berühmtesten Buhlerinnen gehörte, pafst nicht zu der Romanheldin, die nach dem üb- 
lichen Schema ein sehr tugendhaftes Wesen sein muls. Es scheint also, dafs auch schon in diesem Roman von der ursprünglichen Sage nicht viel mehr als die Namen übrig geblieben sind. Vgl. oben S. 257.

In der Art der Darstellung ähnelt das Fragment vielfach den andern Romanen. Den grölsten Teil nimmt eine pathetische Rede des Liebhabers ein, und kurz vorher wird, wie Kaibel und Robert erkannt haben, ein epideiktischer Vortrag eines Fremden gestanden haben. Also auch hier wieder wie schon im ältesten Roman von Ninos und wieder in den späteren die Vorliebe für wohlgesetzte Reden. Dals auch dem Inhalt nach zahlreiche Fäden diesen Roman mit den anderen verbinden, soll kurz hier dargelegt werden.

In dem vorliegenden Fragment, das offenbar dem Anfang des Romanes angehört, ist Metiochos als der spröde Jüngling gezeichnet, wie ihn auch die anderen Romane so gern schildern, um ihn dann in das Gegenteil umschlagen zu lassen. Vgl. Rohde S. 146/7. Wir werden in eine lebhafte Debatte hineingeführt, die sich, wie so häufig in dieser Litteratur, um das Wesen des Eros dreht. Nachdem jener Fremdling die herrschende mythologische Auffassung vorgetragen hat, wendet sich Metiochos, der den Eros selbst noch nicht kennen gelernt hat, in Gegenwart der Parthenope mit rationalistischen Gründen dagegen. Kaibel und Robert, die diese Situation erkannt haben, denken sich als Schauplatz „vielleicht eine Rhetorenschule für beiderlei Geschlechter". Man könnte sich auch eine andere Scenerie denken, die in den erhaltenen Romanen öfter begegnet: das Gespräch hönnte an ein Bild des Eros anknüpfen, wozu der Roman des Eustathios (II 7 ff.) die beste Parallele bieten würde. Da steht der Held, der ebenso wie Metiochos noch nichts von Liebe weifs, vor einem Erosbilde und philosophiert mit seinem in die Liebe schon eingeweihten Freunde über das Wesen der Gottheit. ${ }^{1}$ ) Auch auf die Einleitung zum Roman des Achilles Tatius und auf das Prooemium des Longus könnte man hinweisen.

Doch mag die Situation im Parthenope-Roman sein wie sie will, jedenfalls ist von Interesse, dals der Grundgedanke hier und bei dem Byzantiner derselbe ist. Auch bei Eustathios nimmt der Jüngling aus rationalistischen Gründen Anstofs an den hergebrachten mythologischen

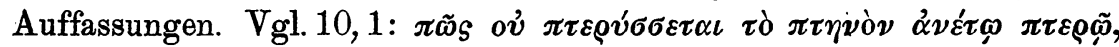

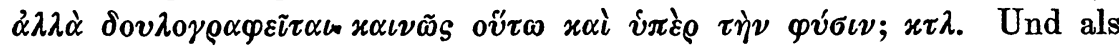
der Freund ihm dann von der allbezwingenden Macht des Eros spricht,

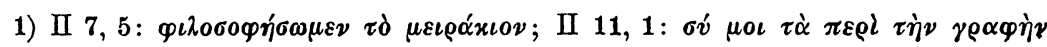

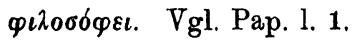


bricht er die Unterhaltung mit den ahnungsvollen Worten ab: $M \eta \delta^{\grave{\varepsilon}}$

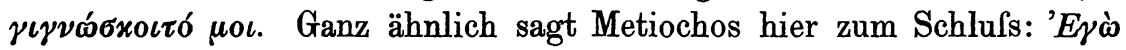

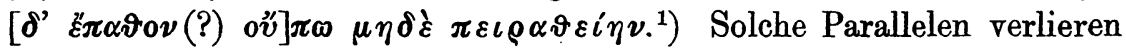
dadurch nicht, dafs wir sie bei einem Byzantiner finden. Gerade bei diesen, denen die Erfindung neuer Situationen und Gedanken am wenigsten zuzutrauen ist, werden wir oft auf das Gut vergangener Jahrhunderte zu stofsen erwarten dürfen. Derselbe Gedanke kehrt übrigens etwas versteckt auch im Prooemium zu Longus' Roman

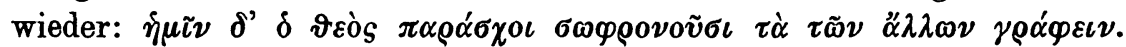

Während bei Eustathios der Jüngling dem Freunde nur verwunderte Fragen entgegenhält, greift Metiochos die Auffassung des Fremden in ihren einzelnen Punkten scharf an. In der Ausführung bietet dieser Roman, wie zu erwarten, Neues, Originelles, doch die Grundgedanken sind auch den anderen Romanen bekannt. So erklärt Metiochos es für lächerlich, dafs Eros ewig ein Knabe geblieben sein solle. ${ }^{2}$ ) Dafs Eros in Wirklichkeit sehr alt sei und nur jung erscheine, ist ein auch bei Longus

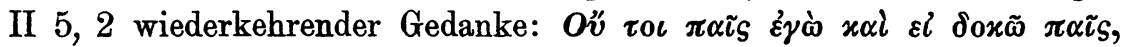

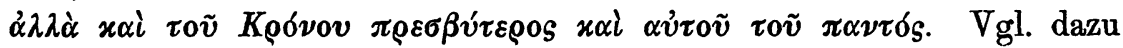
Plato, Sympos. p. 195 B f. ${ }^{3}$ ) Vgl. auch Lucian, dial. deor. 2, 1 und Apul. Metam. V 31. Wenn Metiochos zum Sehlufs eine rein natur-

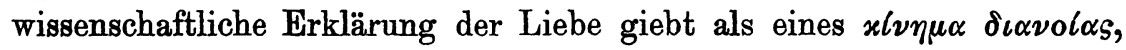
das durch $\pi \alpha$ óvos entstehe und durch $\sigma v v \eta \dot{v} \varepsilon \iota \alpha$ wachse, so ähnelt er in dieser Gottesleugnung dem spröden ' $A \beta \rho о x o ́ \mu \eta s$ in den Ephesiaca

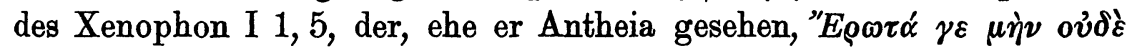

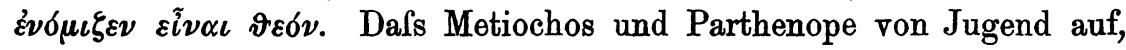
wie es scheint, in Freundschaft miteinander aufwuchsen, ohne zunächst von Liebe etwas zu wissen, erinnert an Daphnis und Chloë bei Longus, während in den meisten anderen Romanen der Jüngling erst später das Mädchen erblickt und dann sofort in Liebe entbrennt.

So finden wir auch diesen Roman, der wohl spätestens um 100 n. Chr., vielleicht viel früher, entstanden ist, durch mancherlei Parallelen mit den anderen Vertretern dieser Gattung verknüpft ${ }^{4}$ ), während sich anderes als originell erweist.

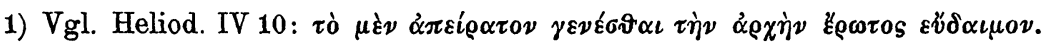

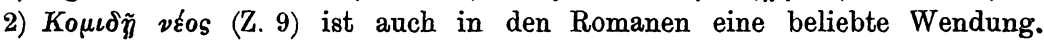
Vgl. Longus IV 24, 1.

3) Dafs manche Gedanken und Ausdrücke in den Romanen auf Plato zurückgehen, hat speziell für Achilles Tatius gezeigt Andreas Stravoskiadis, Ach. Tat. ein Nachahmer des Plato, Aristoteles, Plutarch und Aelian. Erl. Diss. 1889.

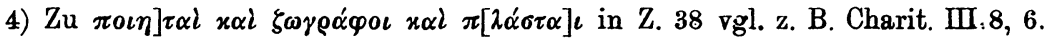


Endlich hat Mahaffy in den Rendiconti della Reale Accademia dei Lincei vom 21. Februar 1897 ein Fragment ediert, das er, wie mir scheint, mit Recht als Bruchstück aus einem Roman bezeichnet hat. Eine vortreffliche Erklärung des Textes verdanken wir Otto Crusius (Beilage zur Allgem. Zeitung 1897 Nr. 145, 3. Juli).

Mahaffy hat übereinstimmend mit Kenyon und Grenfell die Schrift des Romanes, der auf dem Verso steht, in das II. Jahrh. n. Chr., die Schrift des Recto (Abrechnungen etc.) in das I. Jahrh. n. Chr. gesetzt. Auch ich kann mich auf Grund der beigefügten Photographien dieser Schätzung nur anschliefsen, kann für den zweiten Ansatz sogar einen direkten Beweis erbringen. In der unteren Hälfte des Recto lese ich die Worte: $\Sigma \varepsilon \beta \alpha \sigma \tau o \tilde{v} \Gamma \varepsilon \rho \mu \alpha \nu \iota x о \tilde{v}$ $\Phi \alpha \mu \varepsilon v[\grave{\omega} \vartheta$. Damit kann nur Domitian gemeint sein, und wenn ich nicht irre, passen auch die vorhergehenden Schriftspuren zu $\Delta 0] \mu \underline{\text { c }} \_\alpha \nu 0 \tilde{v}$. Damit ist der terminus post quem für die Romanhandschrift gegeben. Es war ein Irrtum, wenn Crönert oben S. 210 den Papyrus der Ptolemäerzeit zuwies.

Erhalten ist vom Roman eine Kolumne von 60 Zeilen, links und rechts davon einige Schlüsse und Anfänge der benachbarten Kolumnen. Der Text ist in einer an sich leicht lesbaren Kursive geschrieben, ist aber leider durch Lücken und Beschädigungen der Oberfläche derartig verderbt, dafs von den 60 Zeilen bis jetzt nur ganz wenige vollständig und sicher gelesen sind. Viele von Mahaffys Lesungen habe ich auf der Photographie nicht wiedergefunden, ohne doch sagen zu können, was wirklich dasteht. An einigen Stellen bin ich etwas weiter gekommen, aber meine Hoffnung, einen zusammenhängenden Text schaffen zu können, habe ich bald aufgeben müssen. Das wird nur am Original zu machen sein. Hoffen wir, dafs der hochverdiente Herausgeber sich der Mühe unterzieht, den, wie es scheint, in seinem Privatbesitz befindlichen Text nochmals gründlichst zu revidieren. Einstweilen teile ich die kleinen Ergebnisse meiner Nachlese mit.

In der vorhergehenden Kolumne, die Mahaffy ungelesen gelassen

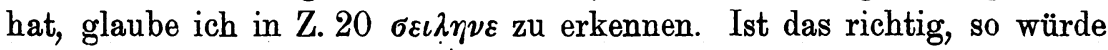
dort ein Mann $\Sigma \varepsilon \iota \lambda \eta \nu o ́ s$ angeredet, also wohl ein Gespräch wiedergegeben sein.

Oberhalb der Hauptkolumne erkenne ich, was sich für die Interpretation als nicht ganz unwichtig ergeben wird, die von zweiter Hand später hinzugefügte Paginazahl $K \Theta$. Wir haben also die $29^{\text {te }}$ Kolumne der gesamten Darstellung vor uns.

Z. 7 sehe ich xảyì̀, darauf vielleicht $\mu o ́ v$ [os? am Schlufs ein Praeteritum mit $\boldsymbol{\nu}$ abschliefsend. Dann beginnt ein neuer Satz: Tô $\nu$ $\delta \grave{\varepsilon}[x] v \beta \varepsilon \rho \nu \eta \tau \tilde{\omega} \nu$. Eine Verbalform wie $\pi \alpha \rho \alpha \iota \nu \rho v ́ v \tau \omega \nu$, die Mahaffy 
vor $\delta \xi$ erwartet, würde nicht passen, weil dann der Artikel hinter $\delta \xi$ zu erwarten wäre. Das Participium mufs vielmehr folgen. Darauf

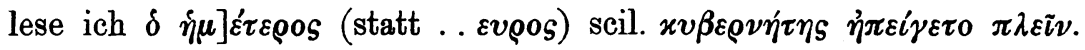

Z. 16 erkenne ich $\pi \rho 0[s] \omega \pi \lambda l \xi \varepsilon \tau o$ statt $\pi .[..] \omega \pi \lambda \iota \xi \varepsilon \tau o$, und zum

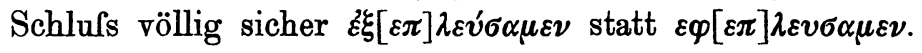

Z. 17 scheint $\dot{\eta} \pi \varrho 0 \vartheta \mu i_{S} \tau \dot{\eta} \nu$ zu stehen, statt $\eta \pi \rho 0 \vartheta \alpha[.] . \tau \tau \varepsilon \iota \nu$. $\Pi \varrho 0 \vartheta \mu l_{S}$ würde vulgäre Schreibung sein, mit einer häufig vorkommenden Metathese, für $\pi 0 \varrho \vartheta \mu i$ s.

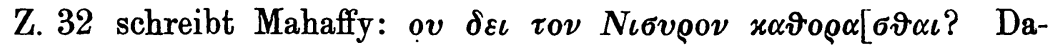
nach übersetzt Crusius "(Wir werden über das kretische Meer dahingetrieben), wo man Nisyros sehen mufs". Befriedigender ist die folgende Lesung, die mir sicher erscheint: ov̉dz์ rend es nicht mehr möglich war, Nisyros (vom Schiff aus) zu sehen“. Daraus lernen wir, dafs die Fahrt von Nisyros ausgegangen war!

Z. $55 \mathrm{f}$. lese und ergänze ich etwa folgendermafsen: $] \lambda \varepsilon$ iò $x \tilde{v} \mu \alpha$.

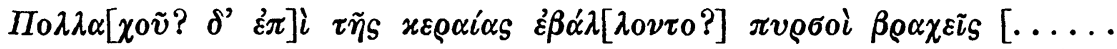

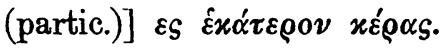

Z. 57 Schlufs steht nicht mit Abbreviatur $\pi \varrho о \sigma \omega \nu v \mu \iota$. Also kann das Folgende nicht zu $\alpha \nu[\alpha \pi \varepsilon \sigma] о \nu \tau \varepsilon s$ ergänzt

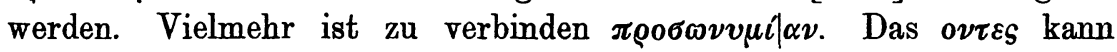
ich nicht sicher erkennen.

Der Inhalt der Kolumne ist kurz folgender: Zwei Personen, von denen eine den Hergang erzählt, nehmen unter „alkyonischen Klagen“ zärtlichen Abschied von einander. Beide verlassen den Hafen, wie wir soeben sahen, von Nisyros, aber auf verschiedenen Schiffen. Ein Unwetter zieht sich zusammen, das allmählich in furchtbaren Sturm mit Blitzen und Regengüssen ausartet, bis schliefslich - wie Crusius so schön gezeigt hat - das Rettung verheirsende St. Elmsfeuer sich auf die Raaen setzt, worauf die Mannschaft niederfällt und betet. Damit bricht der Text ab.

Mahaffy erklärt dies für ein Bruchstück eines griechischen Romanes and verweist auf den grofsen Seesturm bei Achill. Tat. III $1 \mathrm{ff}^{1}$ ) Crusius dagegen möchte es lieber der Brieflitteratur zuweisen, unter Hinweis auf den schönen Synesiusbrief, in dem auch ein Seesturm geschildert

1) Vgl. ferner Heliodor V 27; Charit. III 3, 10 ff.; Eustath. VII 7 ff.; hist. Apoll. c. 11, 12 und dazu Klebs S. 284. Grofse Ähnlichkeit mit unserem Fragment zeigt in vielen Details die Sturmbeschreibung in Ovids schöner Erzählung Jon Keyx und Alkyone, die nach Nikander erzählt sein soll. Auch unsere Darstellung mag in letzter Instanz auf hellẹnistische Vorbilder zurückgehen. 
wird, oder möchte es wegen der Dioskurenepiphanie auch wohl für

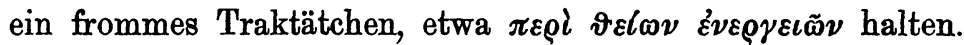

Ich gebe Crusius zu, dafs ein strikter Beweis für die Zugehörigkeit zu den Romanen nicht zu erbringen ist, und dafs die Stilverwandtschaft unseres Fragmentes mit den späteren Romanen, die auch nach Crusius unverkennbar ist, die Frage nicht löst; aber auch die Einwendungen, die er gegen Mahaffys Annahme erhebt, scheinen mir nicht entscheidend zu sein.

Sein erster Einwurf betrifft die Personen: „in dem Roman handelt es sich um ein Liebespaar, hier ist von zwei Freunden oder Kameraden die Rede“. Die Praemisse ist nicht in dem Sinne zuzugeben, dafs nicht auch im Roman ausführlichst von den gemeinsamen Abenteuern zweier Freunde gehandelt werden könnte. Man denke nur an Chariton, bei dem Chaereas und Polycharm auf der Suche nach Kallirrhoë die tollsten Gefahren miteinander bestehen, ja, abgesehen vom Anfang und vom Schlufs tritt Chaereas meist mit dem Freunde zusammen auf, nicht mit der Geliebten. Warum sollte nicht auch hier eine solche Episode erzählt sein? Vielleicht segeln auch hier die beiden Freunde hinaus, um die Geliebte des Einen zu suchen. ${ }^{1}$ ) Doch ich will dieser Vermutung um so weniger nachgehen, als es mir garnicht sicher ist, dafs wir wirklich

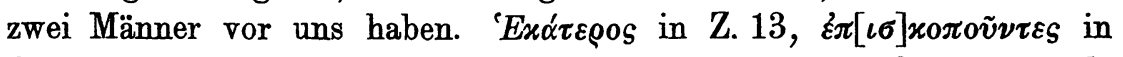

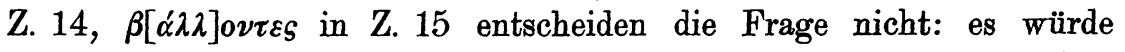
nicht anders heifsen, wenn die andere Person ein Weib wäre. Die Möglichkeit ist jedenfalls bei der jetzigen Textgestalt zuzugeben. ${ }^{2}$ ) Ja ich meine, die Zärtlichkeit des Abschiedes, die Handküsse, die sie sich

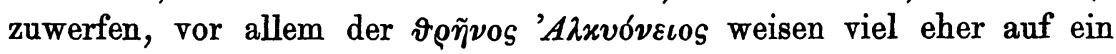
Liebespaar hin. Doch die Frage mufs offen bleiben. Jedenfalls kann nach obigem aus dem Personenstande ein Kriterium gegen den Romancharakter des Fragmentes nicht abgeleitet werden.

Crusius bemerkt ferner, dafs wegen des St. Elmsfeuers, das einen günstigen Ausgang verheifst, der Sturm nicht zu einem Schiffbruch geführt habe, wie das in den Romanen geschehe. Es ist richtig, dals in den Romanen die Stürme oft zum Schiffbruch führen, aber es giebt auch da Fälle, in denen das Schiff schliefslich glücklich landet. Mir fällt z. B. folgende Stelle in der hist. Apoll. c. 39 ein: . . sublatis ancoris altum pelagus petiit iam ad Tyrum reversurus. Qui dum prosperis ventis navigat, subito mutata est pelagi fides, per diversa discrimina

1) So könnte es sich erklären, dafs sie aus demselben Hafen auf verschiedenen Schiffen abfahren. Ähnliches bei Charit. III 3, 8 .

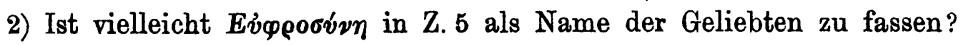


maris iactantur; omnibus deum rogantibus ad Mytilenam civitatem advenerunt. In diesem kurzen Auszug (s. oben S. 260 Anm.) ist zwar der Seesturm nicht ausführlicher beschrieben. Aber wenn man von Tarsos ausfahrend statt nach Tyros nach Mytilene kommt, mufs es doch ein recht bedeutender Sturm gewesen sein - im griechischen Original mag er ausführlich geschildert gewesen $\operatorname{sein}^{1}$ ) -, und doch endet er mit einer glücklichen Landung. Doch hiergegen liefse sich manches einwenden; sicherer beweist Heliodor V 27: da ist ein Seesturm mit grofser Ausführlichkeit geschildert; dennoch erfolgt kein Schiffbruch! Dieses Heliodorkapitel zeigt uns, dafs gelegentlich auch die Romanschriftsteller, wenn sie ihre Helden über das Meer fahren liefsen, diese Gegenheit benutzten, einen Seesturm darzustellen, auch wenn daraus keine Folgen für die Entwicklung der Handlung sich ergeben sollten also aus reiner Freude an der beliebten हैx Hal. rhet. 10,17. Und damit würde auch unser Fragment als Episode innerhalb eines Romanes genügend erklärt sein.

Was andrerseits die positiven Vorschläge von Crusius betrifft (Brief oder Traktat), so gehen sie wohl von der Vorstellung aus, dals unsere Erzählung keinen allzugrofsen Umfang gehabt habe. Sie verlieren daher an Wahrscheinlichkeit, nachdem oben gezeigt ist, dafs die vorliegende Kolumne die $29^{\text {te }}$ ist. So spricht die Seitenzahl für eine breite Darstellung, wie die Romane sie fordern. ${ }^{2}$ )

Vielleicht wird auch diese Hauptfrage klarer zu erkennen sein, wenn erst ein zusammenhängender Text hergestellt sein wird. Einstweilen ist es mir aus den angeführten Gründen wahrscheinlicher, dafs wir einen Roman vor uns haben.

Nachdem der vorstehende Aufsatz in den Druck gegeben war, kam mir die von Fritz Schöll besorgte zweite Auflage des klassischen Werkes von Erwin Rohde zu Gesicht. Leider haben sich eingehendere Untersuchungen über die neuen Romane in seinem Nachlals nicht gefunden. Aus den flüchtigen Randbemerkungen seines Handexemplars

1) Die Worte omnibus deum rogantibus erinnern an das Gebet zum Schlufs unseres Fragmentes. Sie stammen zwar in dieser Form aus dem christlichen Bearbeiter, aber vorher mag ein heidnisches Gebet dort gestanden haben.

2) In dem erhaltenen Fragment erzählt jemand seine eigenen Erlebnisse. Vielleicht war das nur eine Einlage, wie z. B. bei Heliodor sich sehr ausführliche Ich-Erzählungen finden. Es kann aber auch der ganze Roman dieser Person als Erzählung in den Mund gelegt worden sein; dann würden Achilles Tatius, auch Eustathios Parallelen bieten, die gleichfalls einen Ich-Roman geben. 
geht nur so viel hervor, dafs Rohde zwar die Ninos-Geschichte (vgl. S. 577 der 2. Aufl.), aber nicht die Parthenope-Geschichte für einen Roman gehalten hat. In Bezug auf letztere findet sich auf S. 570

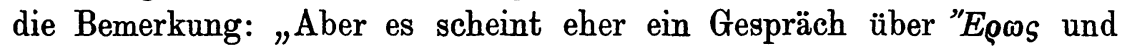

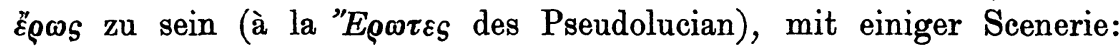
für einen Roman würde das Gespräch kaum passen." Mir scheint aus der obigen Behandlung des Fragmentes, die jch unverändert habe stehen lassen, im besonderen aus der obeñ aufgedeckten Parallele mit Eustathios' Roman doch so viel mit Sicherheit hervorzugehen, dafs das Fragment einem Roman angehören kann. Für die Annahme eines Gespräches in der Art des Pseudolucian scheint mir die Thatsache nicht günstig, dafs Metiochos und Parthenope durch ihre Namen die Erwartung erwecken, dafs sie vom Philosophieren zum Handeln übergehen werden. Doch mögen Berufnere die Frage entscheiden.

Breslau.

Ulrich Wilcken. 